\title{
Precise Self-tuning of Spiking Patterns in Coupled Neuronal Oscillators
}

\author{
I.Y. Tyukin ${ }^{12 *}$, V.B. Kazantsev ${ }^{34}$ \\ ${ }^{1}$ Department of Mathematics, University of Leicester, University Road, LE1 7RH, UK \\ 2 Department of Automation and Control Processes, Saint-Petersburg State \\ Electrotechnical University Prof. Popova str. 5, 197376, Russia \\ ${ }^{3}$ Department of Nonlinear Dynamics, Institute of Applied Physics of RAS \\ Nizhny Novgorod, Russia \\ ${ }^{4}$ Department of Neurodynamics and Neurobiology, University of Nizhny Novgorod \\ Nizhny Novgorod, Russia
}

\begin{abstract}
In this work we discuss and analyze spiking patterns in a generic mathematical model of two coupled non-identical nonlinear oscillators supplied with a spike-timing dependent plasticity (STDP) mechanism. Spiking patterns in the system are shown to converge to a phase-locked state in a broad range of parameters. Precision of the phase locking, i.e. the amplitude of relative phase deviations from a given reference, depends on the natural frequencies of oscillators and, additionally, on parameters of the STDP law. These deviations can be optimized by appropriate tuning of gains (i.e. sensitivity to spike-timing mismatches) of the STDP mechanisms. The deviations, however, can not be made arbitrarily small neither by mere tuning of STDP gains nor by adjusting synaptic weights. Thus if accurate phase-locking in the system is required then an additional tuning mechanism is generally needed. We found that adding a very simple adaptation dynamics in the form of slow fluctuations of the base line in the STDP mechanism enables accurate phase tuning in the system with arbitrary high precision. The scheme applies to systems in which individual oscillators operate in the oscillatory mode. If the dynamics of oscillators becomes bistable then relative phase may fail to converge to a given value giving rise to the emergence of complex spiking sequences.
\end{abstract}

Keywords and phrases: nonlinear phase oscillators, spike-timing dependent plasticity, adaptive control, nonlinear parametrization, convergence, weak attractors, neural oscillators

Mathematics Subject Classification: 92B25, 92C20, 37N25, 37M20

\section{Introduction}

Precise and robust firing activity in neural systems in which clusters of neurons produce time-locked spiking sequences has recently received substantial attention in the literature $[9,10,13,21]$. Despite inherent variability in the dynamics of individual oscillators, relative time lags between spikes in these sequences are robust; the sequences can repeat spontaneously, or they can be generated in response

${ }^{*}$ Corresponding author. E-mail: I.Tyukin@le.ac.uk

(C) EDP Sciences, 2012 
to a certain stimulus. A number of theoretical frameworks have been proposed to explain emergence and persistence of these precise firing patterns with different inter-spike timing, see e.g. [10] and related notions of synchronized chains (synfire chains) and polychronous groups. In these frameworks spike-timing dependent plasticity (STDP), linked to the post-to-presynaptic timing, is advocated as a mechanism that is directly responsible for the emergence of persistent spike sequences within a given topological substrate.

For a pair of synaptically connected cells, STDP stands for a change in synaptic efficacy as a function of timing between pre- and post- synaptic events. If the post-synaptic event occurs within a given interval of time from the onset of the pre-synaptic one then efficacy of synaptic transmission enhances. If, however, the opposite takes place, i.e. a post-synaptic event is followed by pre-synaptic spike, then the efficacy decreases (see Fig. 1). At the level of physical organization, several factors may affect the efficacy of
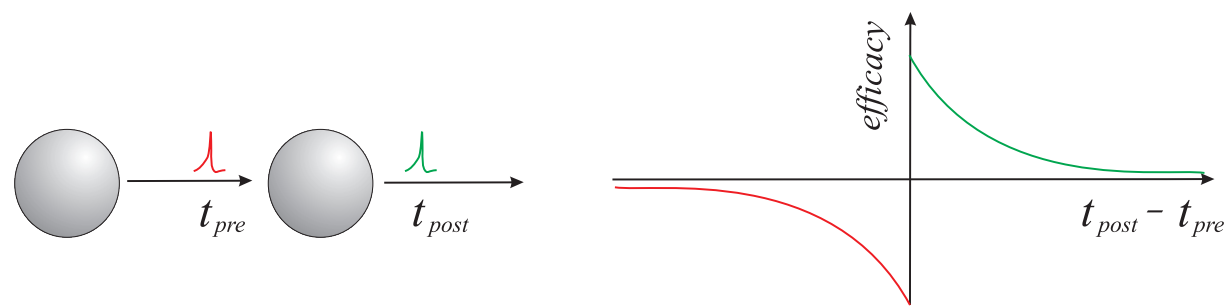

FiguRE 1. Phenomenological description of spike-timing dependent plasticity

signal transmission in the cells [8]. They include: distance between the synapse and the soma, number of connections between neurons, dynamic regime of firing (such as e.g. bursting, when multiple spikes are transmitted in response to single event), long-term potentiation and depression, presence of chemicals and neurotransmitters, and state of the membrane potential relative to the threshold value. At the level of processes, many forms of STDP have been discovered to date [24], and a common knowledge is that STDP is supported by multiple molecular cascades inducing changes in both postsynaptic spines and in presynaptic terminals. Calcium flux through NMDA receptors located in spines [17] is an example of mechanisms directly responsible for postsynaptic changes. In this mechanism, excitatory postsynaptic potentials preceding back-propagating action potentials elicit calcium influx through postsynaptic NMDA receptors. Higher calcium concentration, in turn, facilitates evoking of postsynaptic spikes in response to the presynaptic ones. Changes in presynaptic terminals are observed, for example, in the hippocampal mossy fiber synapses [18]. STDP-like phenomena can also occur due to the modulation of synaptic transmission by endocannabinoid-mediated retrograde cascades. These cascades, once activated, trigger the activation of presynaptic receptors $[3,19]$.

Large diversity of the ways in which STDP may manifest itself in empirical observations has lead to a broad range of mathematical models of the phenomenon. These models, although phenomenological, are widely used in computational and theoretical studies (see e.g. $[5,12,25,28]$ ). In the majority of these models the principal factor determining synaptic efficacy is the synaptic weight. The latter is described by a dynamic variable of which the value changes in response to post-to-presynaptic spike timing. Increments/decrements of the weights are often associated with LTP/LTD respectively. One of the outcomes of such activity-dependent modifications of the synaptic weights is that connections between individual cells may grow or decay over time by a relatively large amount. This facilitates emergence of neuronal clusters that fire together, up to a tolerance margin.

Despite abundance of reports suggesting a possible role of the classical connection-modulating STDP in the formation of spiking patterns in networks of interconnected cells, the question is whether these patterns (including timing between events) are fixed by the system's parameters or they can vary in a broad set depending on stimulation. Previous study [14] showed that achieving arbitrary phase lags between spiking events in a pair of non-identical synaptically connected neural oscillators may not always 
be possible in case of conventional non-dynamic coupling. Yet, this goal could still be reached in a system in which internal states of the oscillators are made dependent on timing between post- and pre-synaptic spikes. The results were shown to hold for a generic system comprised of the pair of phase oscillators, and validity of the analysis was confirmed for the pair of Rowat-Selverston oscillators [22]. It is unclear, however, if the phenomenon would persist for another model neurons.

In this work we extend the results of [14] to the Hodgkin-Huxley model and also assure robustness of the discussed adaptation scheme. We confirm that if the model operates in the oscillatory regime then dynamics of the coupled system (including the ability to reproduce spiking patterns with a broad range of phase lags) remains qualitatively similar to the previously considered case of Rowat-Selverston oscillators. There are, however, marked differences too. These differences are due to that Hodgkin-Huxley equations may exhibit not only pure oscillatory dynamics but also they may operate in the bistable regime in which two stable attractors, a limit cycle and an equilibrium, co-exist in the system's state space. We show that if oscillators are in the bistable mode then relative phase may fail to converge to a given value. Complex spiking sequences will instead be produced in the system.

The paper is organized as follows. Section 2 presents mathematical description of the considered configurations of the coupled neural oscillators. In Section 3 we review theoretical analysis of asymptotical properties and limitations of the system with regards to the range of phase locking values. We also show how these limitations can be overcome by adding a simple adaptation mechanism into the original system. The adaptation mechanism can be made robust to unmodeled dynamics and external perturbations. Section 4 contains results of numerical study of the model, and Section 5 concludes the paper.

\section{Model}

\subsection{Topology of connections and model of STDP}

Consider a phenomenological model of synaptic transmission in a pair of spiking neuronal oscillators supplied with an adaptive STDP regulatory mechanism. A diagram describing this mechanism is schematically presented in Fig. 2. The diagram shows two possible ways in which the timing of spikes may influence state of synaptic coupling. The first alternative is illustrated in Fig. 2A. Timing of pre- and post-synaptic spikes is affecting the state of the presynaptic neuron. Such change of the neuron's state is accounted for in the model by a phenomenological variable $z_{\text {pre }}$. Increasing/decreasing the value of $z_{\text {pre }}$ facilitates/depresses transmission of stimuli, respectively. Such spike-timing-modulated signal transmission in the model acts as a feedback relating timing of pre-to-post synaptic spikes with the neuron's excitability parameter $z_{\text {pre }}$.

Dynamics of this phenomenological variable, $z_{\text {pre }}$, is driven by an STDP function curve of which the shape depends on specific molecular mechanisms. Here, for illustrative and computational purposes, we model this curve by a simple function resembling a truncated sinusoid (Fig. 2C). This STDP curve determines dependence of $z_{\text {pre }}$ on relative time differences between post- and presynaptic spikes (e.g. relative spiking phase). These relative time differences are denoted by $\Phi$.

In addition to the relative spiking phase, $\Phi$, the model accounts for an optional phase offset, $\Phi_{c}$. The latter can be added to or subtracted from the value $\Phi$. The origins of this extra variable are many: it can account e.g. for the influence of delays inherent to signal transmission in neural circuits; it may also model external inputs to the presynaptic neuron. In the context of our present work we will view variable $\Phi_{c}$ as a reference relative phase: the relative phase between spikes which is to be attained asymptotically. In addition to the STDP curve and the phase offset $\Phi_{c}$, we also introduce a regulatory parameter $\lambda_{\text {pre }}$. This extra parameter determines the baseline to which the values of $z_{\text {pre }}$ relax in absence of stimulation. In the model it accounts for small and relatively slow fluctuations of extracellular medium. One can speculate that these fluctuations could be related to glia and matrix influence on synapses - the subject which has been discussed in empirical studies $[4,7,15]$. The latter fluctuations affect the function of STDP and thus they can also be related to metaplasticity [1]. 

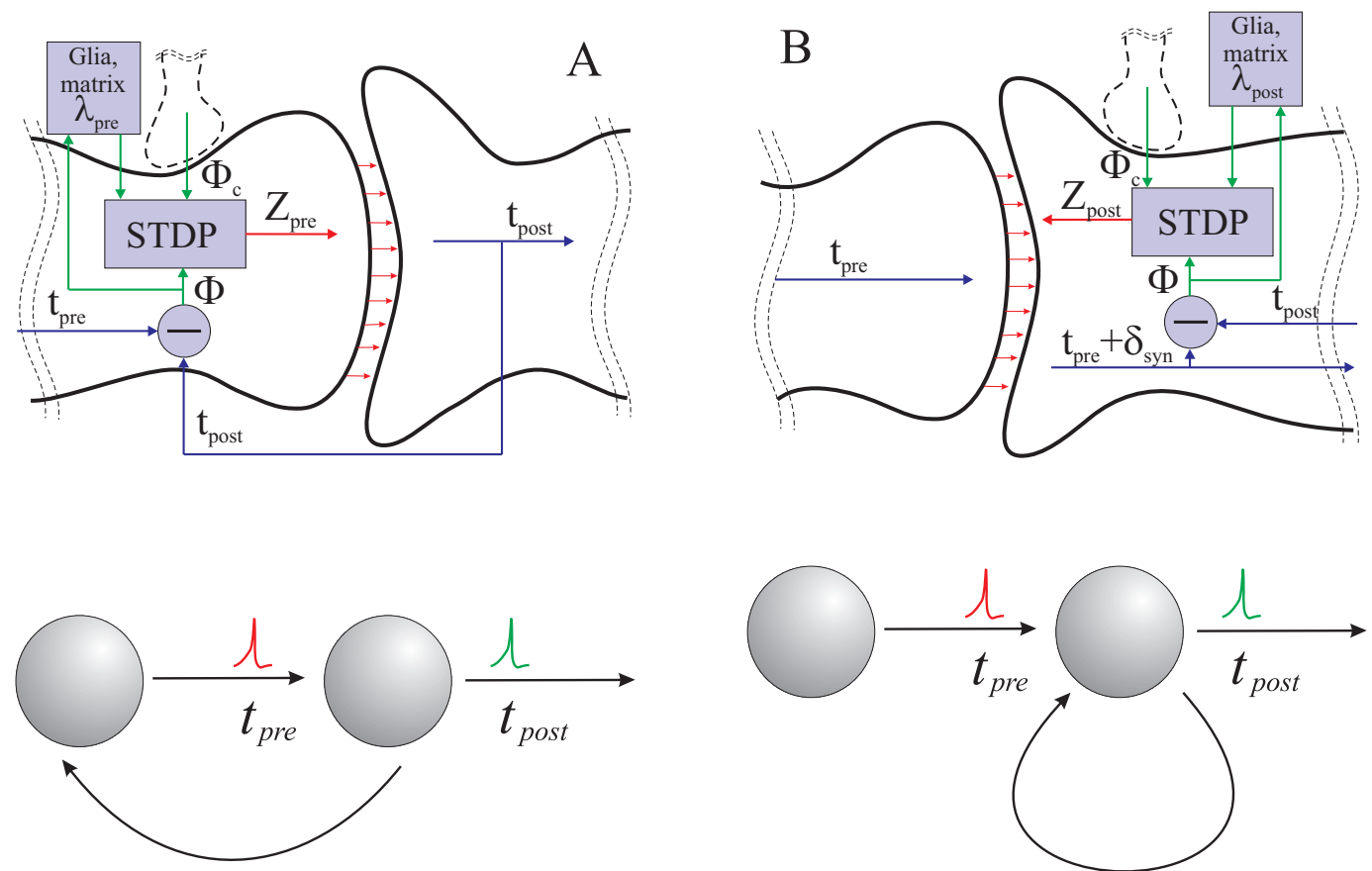

STDP feedback

STDP feedback

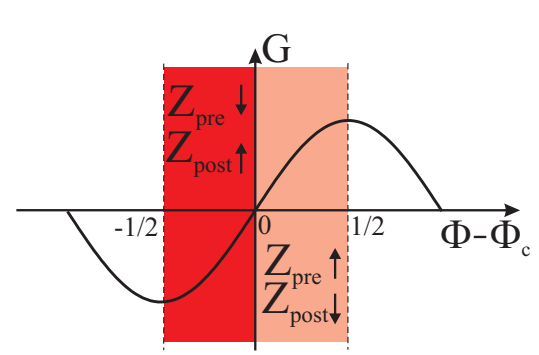

Figure 2. Schematic representation of the adaptive STDP phase locking. Timing between the postsynaptic and the presynaptic spikes is modeled by the spiking phase, $\Phi$. The difference between $\Phi$ and $\Phi_{c}$, where $\Phi_{c}$ is some reference value that might be induced by another regulatory inputs, activates the feedback mechanism, "STDP"; the latter activates molecular cascades changing the state, denoted by $z$, of the presynapse and/or postsynapse. Direct STDP feedback is modulated by fluctuations of extracellular medium, $\lambda$ (e.g. the metaplasticity), giving rise to the adaptation, i.e. fine tuning of the phase-locked state. A: Presynaptic STDP feedback. B: Postsynaptic STDP feedback. C: STDP curves used in simulations. Positive half-period of the $G$-function indicates potentiation by the increase of presynaptic frequency and/or depression by the decrease of postsynaptic frequency.

The second alternative is illustrated in Fig. 2B. Here spike-timing affects the state of the postsynaptic neuron. Spikes arriving to terminals of the presynaptic neuron cause the release of a neurotransmitter. The neurotransmitter reaches the postsynaptic neuron, and this triggers generation of postsynaptic po- 
tentiation (PSP) with latency time $\delta_{s y n} \ll T_{s}\left(T_{s}\right.$ is the characteristic time scale of the spike train, e.g. the period of oscillations). In this model PSP, in turn, triggers generation of the response spike (e.g. action potential). The latter event is then detected in the postsynaptic terminal via a chemically or electrically back-propagating signal. Similarly to the previous (presynaptic) case there is a state variable $z_{\text {post }}$ whose increase or decrease facilitates potentiation or depression, respectively. Other parameters of this mechanism such as $\Phi_{c}$ and $\lambda_{\text {post }}$ are similar to the case discussed in the first alternative.

Let us now formulate the STDP models discussed above mathematically. Presynaptic STDP feedback (shown in Fig. $2 \mathrm{~A}$ ) is governed by the following equations:

$$
\left\{\begin{array}{l}
\frac{d z_{\text {pre }}}{d t}=\alpha_{\text {pre }}\left(I_{\text {pre }}-z_{\text {pre }}\right)-k_{\text {pre }} G(\Phi)+\lambda_{\text {pre }} \\
z_{\text {post }}=I_{\text {post }} .
\end{array}\right.
$$

Similarly, postsynaptic STDP has the form:

$$
\left\{\begin{array}{l}
\frac{d z_{\text {post }}}{d t}=\alpha_{\text {post }}\left(I_{\text {post }}-z_{\text {post }}\right)-k_{\text {post }} G(\Phi)+\lambda_{\text {post }}, \\
z_{\text {pre }}=I_{\text {pre }} .
\end{array}\right.
$$

In essence, Eqs. (2.1) and (2.2) are additional currents in the presynaptic and postsynaptic neurons, respectively. The current are dependent on spike-timing. Parameters $\alpha_{\text {pre }}, \alpha_{\text {post }}$ stand for the time scales of the polarization's relaxation, and $G(\Phi)$ accounts for the STDP curve. Parameters $k_{\text {pre }}, k_{\text {post }}$ are gains. Function $G$ is in the right-hand side of (2.1), (2.2) is assumed to be bounded, sufficiently smooth, and " 1 "-periodic. In particular, the following is supposed to hold:

$$
\begin{aligned}
& G(\Phi) \in \mathcal{C}^{2} \\
& G(\Phi)=G(\Phi+1) \\
& \frac{d G}{d \Phi}\left(\Phi=\Phi_{c}\right)>0 .
\end{aligned}
$$

Variable $\Phi_{c}$ in $(2.3)$ is the reference phase, $0<\Phi_{c}<1$. In the present work, for simplicity, we select the function $G$ as follows:

$$
G(\Phi)=\sin \left(2 \pi\left(\Phi-\Phi_{c}\right)\right) .
$$

\subsection{Models of neuronal oscillators}

In the present work two different yet related sets of equations were chosen to represent electrical activity in the neurons (depicted as gray shaded circles in Fig.2). The first set is the standard Hodgking-Huxley equations, and the second is a computationally efficient reduction of these equations, known as the RowatSelverston model. The choice of models is motivated by that the later is widely used in computational neuroscience in the context of synchronization [2], whereas the former is the model of choice when biological realism is required.

\subsubsection{Hodgkin-Huxley equations}

The Hodgkin-Huxley-based equations [11] of the coupled system are:

$$
\begin{aligned}
\dot{V}_{\text {pre }}= & -z_{\text {pre }}-\Delta I-\left[g_{N a} m_{\text {pre }}^{3} h_{\text {pre }}\left(V_{\text {pre }}-E_{N a}\right)+g_{K} n_{\text {pre }}^{4}\left(V_{\text {pre }}-E_{K}\right)\right. \\
& \left.+g_{L}\left(V_{\text {pre }}-E_{L}\right)\right] \\
\dot{m}_{\text {pre }}= & \left(1-m_{\text {pre }}\right) \alpha_{m}\left(V_{\text {pre }}\right)-m_{\text {pre }} \beta_{m}\left(V_{\text {pre }}\right) \\
\dot{n}_{\text {pre }}= & \left(1-n_{\text {pre }}\right) \alpha_{n}\left(V_{\text {pre }}\right)-n_{\text {pre }} \beta_{n}\left(V_{\text {pre }}\right) \\
\dot{h}_{\text {pre }}= & \left(1-h_{\text {pre }}\right) \alpha_{h}\left(V_{\text {pre }}\right)-h_{\text {pre }} \beta_{h}\left(V_{\text {pre }}\right) \\
\dot{V}_{\text {post }}= & -z_{\text {post }}-\left[g_{N a} m_{\text {post }}^{3} h_{\text {post }}\left(V_{\text {post }}-E_{N a}\right)+g_{K} n_{\text {pre }}^{4}\left(V_{\text {post }}-E_{K}\right)\right. \\
& \left.+g_{L}\left(V_{\text {post }}-E_{L}\right)\right]-I_{\text {syn }}\left(V_{\text {post }}, V_{\text {pre }}\right) \\
\dot{m}_{\text {post }}= & \left(1-m_{\text {post }}\right) \alpha_{m}\left(V_{\text {post }}\right)-m_{\text {post }} \beta_{m}\left(V_{\text {post }}\right) \\
\dot{n}_{\text {post }}= & \left(1-n_{\text {post }}\right) \alpha_{n}\left(V_{\text {post }}\right)-n_{\text {post }} \beta_{n}\left(V_{\text {post }}\right) \\
\dot{h}_{\text {post }}= & \left(1-h_{\text {post }}\right) \alpha_{h}\left(V_{\text {post }}\right)-h_{\text {post }} \beta_{h}\left(V_{\text {post }}\right)
\end{aligned}
$$


where

$$
\begin{gathered}
E_{K}=-12, E_{N a}=120, E_{L}=10.6, g_{K}=36, g_{N a}=120, g_{L}=0.3 \\
\alpha_{n}(V)=0.01 \frac{10-V}{e^{\frac{10-V}{10}}-1}, \alpha_{m}(V)=0.1 \frac{25-V}{e^{\frac{25-V}{10}}-1}, \alpha_{h}(V)=0.07 e^{\frac{-V}{20}} \\
\beta_{n}(V)=0.125 e^{\frac{-V}{80}}, \beta_{m}(V)=4 e^{\frac{-V}{18}}, \beta_{h}(V)=\frac{1}{e^{\frac{30-V}{10}}+1} .
\end{gathered}
$$

Subscripts pre,post in (2.8) label variables governing dynamics of presynaptic and postsynaptic neurons, respectively. Variables $V_{\text {pre }}, V_{\text {post }}$ stand for the corresponding membrane potentials, and $m_{\text {pre }}, m_{\text {post }}, h_{\text {pre }}, h_{\text {post }}, n_{\text {pre }}, n_{\text {post }}$ are variables gating sodium and potassium currents in pre- and postsynaptic neurons respectively. Synaptic current, $I_{\text {syn }}$ is implemented in accordance with the following instantaneous synaptic transmission model:

$$
I_{\text {syn }}\left(V_{\text {post }}, V_{\text {pre }}\right)=g_{\text {syn }} S_{\infty}\left(V_{\text {pre }}\right) \cdot\left(V_{\text {post }}-V_{\text {syn }}\right),
$$

where $g_{\text {syn }}$ is the maximal synaptic conductance reflecting synaptic strength. Function

$$
S_{\infty}\left(V_{\text {pre }}\right)=\frac{1}{1+\exp \frac{\Theta_{\text {syn }}-V_{\text {pre }}}{k_{\text {syn }}}}
$$

defines the amount of available neurotransmitter, and parameters $\Theta_{s y n}$ and $k_{s y n}$ characterize the midpoint and slope of synaptic activation, respectively. Parameter $V_{\text {syn }}$ is associated with the synaptic reversal potential; it controls the sign of synaptic currents induced by spikes at the presynaptic neuron. In this model, the synapse is excitatory, $V_{\text {syn }}=0 \mathrm{mV}$.

Let us illustrate first the dynamics of uncoupled oscillators, e.g. $g_{s y n}=0$. For simplicity, we set $z_{\text {pre }}=z_{\text {post }}=0$ and consider the dynamics of the presynaptic neuron depending on $\Delta I$ playing the role of an external current controlling the depolarization level. Figure 3 illustrates monotonic dependence of oscillation frequency on $\Delta I$. The oscillation appears from saddle-node limit cycle bifurcation at critical point $I_{S N}$. There is bistability region between $I_{S N}$ and $I_{A H}$ where stable limit cycle coexists with stable fixed point. Saddle-type limit cycle whose manifolds divide attraction basins disappears at the second critical point $I_{A H}$ via subcritical Andronov-Hopf bifurcation and the dynamics become true oscillatory.

\subsubsection{Rowat-Selverston equations}

The Rowat-Selverston model can be expressed as follows [22]:

$$
\left\{\begin{array}{l}
\tau_{m} \frac{d V_{\text {pre }}}{d t}=I_{\text {fast }}\left(V_{\text {pre }}\right)-w_{\text {pre }}-z_{\text {pre }}-\Delta I \\
\tau_{w}\left(V_{\text {pre }}\right) \frac{d w_{\text {pre }}}{d t}=w_{\infty}\left(V_{\text {pre }}\right)-w_{\text {pre }} \\
\tau_{m} \frac{d V_{\text {post }}}{d t}=I_{\text {fast }}\left(V_{\text {post }}\right)-I_{\text {syn }}\left(V_{\text {post }}, V_{\text {pre }}\right)-w_{\text {post }}-z_{\text {post }} \\
\tau_{w}\left(V_{\text {post }}\right) \frac{d w_{\text {post }}}{d t}=w_{\infty}\left(V_{\text {post }}\right)-w_{\text {post }} \\
z_{\text {pre }}=I_{\text {pre }}, z_{\text {post }}=I_{\text {post }} .
\end{array}\right.
$$

As before, subscripts pre, post in (2.8) label variables governing dynamics of presynaptic and postsynaptic neurons, respectively. Variables $V_{\text {pre }}, V_{\text {post }}$ stand for the corresponding membrane potentials. Parameters $I_{\text {pre }}, I_{\text {post }}$ model constant currents determining equilibrium depolarization levels; $\Delta I$ is the difference in depolarization (hence, natural spiking frequencies) between two neurons. The function

$$
I_{\text {fast }}(V)=-V+\tanh \left(g_{\text {fast }} V\right)
$$




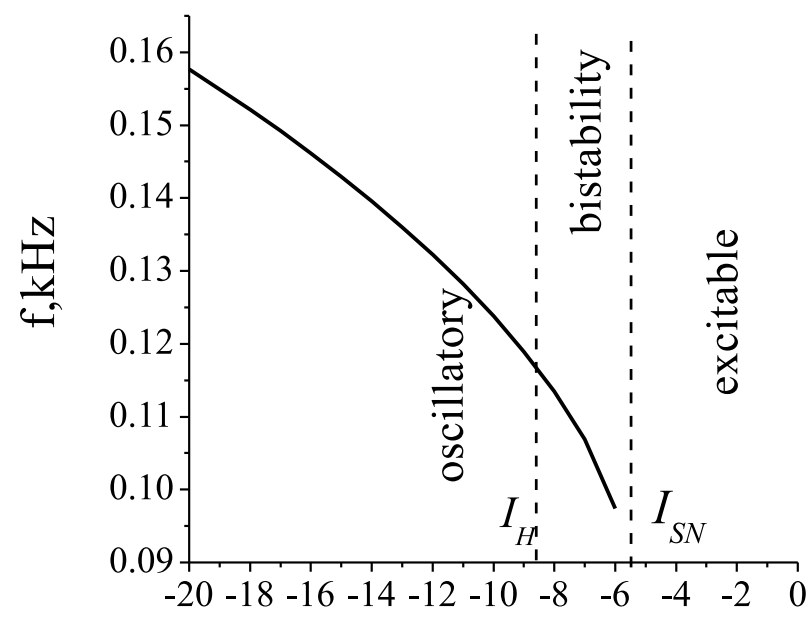

$\Delta \mathrm{I}, \mu \mathrm{A} / \mathrm{cm}^{2}$

Figure 3. Spike frequency dependence on the depolarization level, $\Delta I$, in single Hodgkin-Huxley oscillator

\begin{tabular}{|c|c|}
\hline Parameter & Values \\
\hline$I_{\text {pre }}, I_{\text {post }}, \Delta I$ & $0.5,0.5,[-1.1 \div 0.2]$ \\
\hline$g_{\text {fast }}, g_{\text {slow }}$ & $2.0,2.0$ \\
\hline$\tau_{m}, \tau_{1}, \tau_{2}, k_{\tau}$ & $0.16,5.0,50.0,0.05$ \\
\hline$V_{\text {syn }}, \Theta_{\text {syn }}, k_{\text {syn }}, g_{\text {syn }}$ & $1.0,0.0,0.16,[0.0 \div 1.0]$ \\
\hline
\end{tabular}

TABLE 1. Parameters of model (2.8)

models fast currents across cell membrane, and $g_{\text {fast }}$ is the conductance of the fast voltage-dependent inward current, Variables $w_{\text {post }}, w_{\text {pre }}$ are the slow recovery variables, and $w_{\infty}(V)=g_{\text {slow }} V$ is the voltagedependent activation function; $g_{\text {slow }}$ is the corresponding conductance. Time scales of the spikes are determined by parameter $\tau_{m}>0$ and the function

$$
\tau_{w}(V)=\tau_{2}+\frac{\tau_{1}-\tau_{2}}{1+\exp \frac{-V}{k_{\tau}}} .
$$

The function $\tau_{w}(V)$ is the voltage dependent characteristic time of the slow current, and $\tau_{1}, \tau_{2}, k_{\tau}$ are parameters. We consider the case when $\tau_{1,2} \gg \tau_{m}$ and $\tau_{w}(V) \gg \tau_{m}$, and $\tau_{2}>\tau_{1}$. This ensures that duration of individual spikes is small relative to the inter-spike intervals.

Synaptic current variable, $I_{\text {syn }}$, is defined as in (2.6). The values of all relevant parameters of the model are provided in Table 1 . When $g_{\text {syn }}=0$ pre- and post-synaptic oscillators are uncoupled, both producing sequences of pulses with constant, albeit different, firing rate. 
The oscillation in each uncoupled compartment appear through the saddle-node limit cycle bifurcation $[6,23]$. In terms of Eqs. (2.8), such bifurcation occurs when parameter $\Delta I$ reaches some critical value. This mimics depolarization of the membrane by a constant current injection. Dependence of the spiking rates on the depolarization levels is similar to one obtained for the Hodkin-Huxley model (Fig. 4). In Fig.4, labels $I_{1}$ and $I_{2}$ mark maximal and minimal values of $\Delta I$ for which the dynamics of both

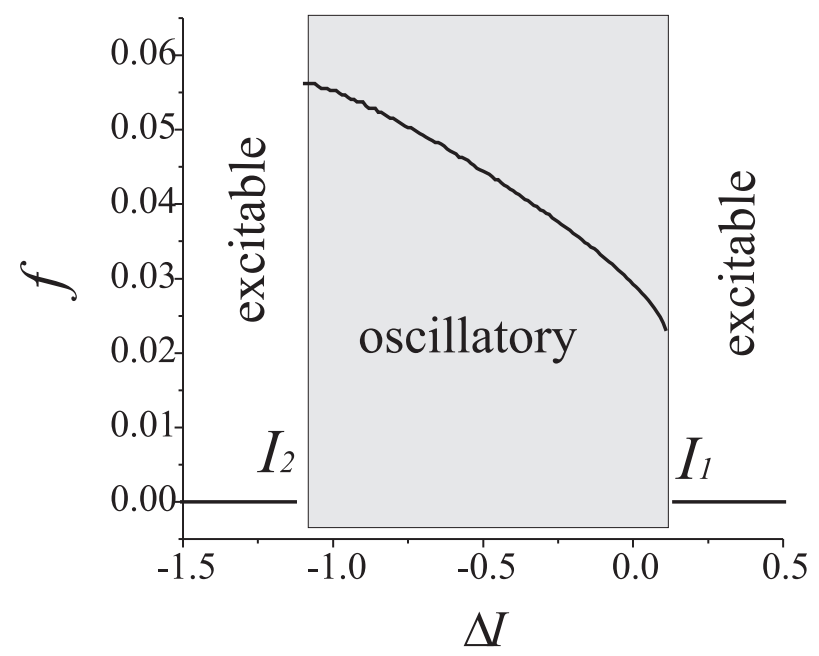

FiguRE 4. Spike oscillation frequency (e.g. natural frequency) as a function of the level of depolarization in single neuron model described by Eqs. (2.8). The values of frequency $f$ are computed for the dimensionless model.

compartments is oscillatory. If the values of $\Delta I$ are outside of this interval then the system is in the excitable mode $^{1}$. If $\Delta I$ is within the interval $\left[I_{2}, I_{1}\right]$ then the frequency curve, $f(\Delta I)$, is a strictly monotone and continuous function. Thus in this interval there is a one to one correspondence between the depolarization parameter $\Delta I$ and the spike firing rate, $f$. Note, that in contrast with Hodgkin-Huxley model the interval of bistability is negligibly small in the Rowat-Selverston model and can be neglected in further consideration.

\section{Analysis}

\subsection{Relative phase dynamics and stabilizing effect of STDP}

For the sake of simplicity in this section we will consider the case when individual spikes are generated by (2.8) and, furthermore, will focus on the configuration in which only one of the two coupled phase oscillators, e.g. postsynaptic, is supplied with the STDP-type mechanism. We will also assume that if the value of $z_{\text {post }}$ is fixed (within an interval) then both oscillators operate in a vicinity of asymptotically stable limit cycles. In addition, we will suppose that the values of $g_{\text {syn }}$ are negligibly small so that we can investigate stabilizing effects of STDP in the combined system independently from effect of phase pulling due to the instantaneous synaptic coupling between oscillators [8].

Under the assumptions above, phase of the oscillations in $V_{\text {pre }}, w_{\text {pre }}$-system (see e.g. [20]) can be defined as a function $\phi_{\text {pre }}\left(V_{\text {pre }}, w_{\text {pre }}, \Delta I\right)$ such that

$$
\frac{\partial \phi_{\text {pre }}}{\partial V_{\text {pre }}} \dot{V}_{\text {pre }}+\frac{\partial \phi_{\text {pre }}}{\partial w_{\text {pre }}} \dot{w}_{\text {pre }}=\omega_{0}\left(I_{\text {pre }}+\Delta I\right)
$$

\footnotetext{
${ }^{1}$ It is necessary to mention here that in principle there are very narrow intervals to the left of $I_{2}$ and to the right of of $I_{1}$ in which unstable limit cycle exists. These are not shown in the figure.
} 
where $\omega_{0}\left(I_{\text {pre }}+\Delta I\right)$ is the natural frequency of oscillations. Since the frequency of oscillations decreases with $\Delta I$ (see Fig. 4), the function $\omega_{0}(\cdot)$ is strictly monotone and non-increasing. Moreover, for a technical reason we will assume that the function $\omega_{0}(\cdot)$ is differentiable or that it can be approximated by a differentiable function in the domain of interest.

Similarly, if the value of $z_{\text {post }}$ is fixed, phase of the oscillations in $V_{\text {post }}, w_{\text {post }}$-system can be expressed as a function $\phi_{\text {post }}\left(V_{\text {post }}, w_{\text {post }}, z_{\text {post }}\right)$ such that

$$
\frac{\partial \phi_{\text {post }}}{\partial V_{\text {post }}} \dot{V}_{\text {post }}+\frac{\partial \phi_{\text {post }}}{\partial w_{\text {post }}} \dot{w}_{\text {post }}=\omega_{0}\left(z_{\text {post }}\right)
$$

Notice that if the value of $z_{\text {post }}$ is allowed to vary and $\left|\dot{z}_{\text {post }}\right|$ is sufficiently small, then $\dot{\phi}_{\text {post }} \simeq \omega_{0}\left(z_{\text {post }}\right)$ provided that $\left|\omega_{0}\left(z_{\text {post }}\right)\right| \gg\left|\dot{z}_{\text {post }}\right|$. The latter inequality reflects that the frequency of oscillations is much higher than the time constant of the STDP. In what follows we will consider the case when this asymptotic holds.

Taking (3.1), (3.2) into account we can conclude that dynamics of the relative phase, $\varphi$, is to satisfy the following equation

$$
\dot{\varphi}=\omega_{0}\left(z_{\text {post }}\right)-\omega_{0}\left(I_{\text {pre }}+\Delta I\right)
$$

Suppose that the variable $\Phi$ in (2.2) evolves according to the following rule:

$$
\Phi_{i}=\frac{t_{\text {post }}(i)-t_{\text {pre }}(i)}{T_{\text {pre }}}
$$

where $t_{\text {post }}(i), t_{\text {pre }}(i)$ are the time instances of postsynaptic and corresponding presynaptic events. Denoting $\omega_{0}\left(z_{\text {post }}\right)-\omega_{0}\left(I_{\text {pre }}+\Delta I\right)=\omega-f\left(z_{\text {post }}\right)$, where $f(\cdot)$ is a continuous, locally Lipschitz, strictly monotone non-decreasing function, and $\omega \in \mathbb{R}$ is the frequency de-tuning parameter, we arrive at the following system of equations:

$$
\begin{aligned}
\dot{\varphi} & =\omega-f\left(z_{\text {post }}\right) \\
\dot{z}_{\text {post }} & \left.=\alpha_{\text {post }}\left(I_{\text {post }}-z_{\text {post }}\right)+k_{\text {post }} G(\sigma(\varphi, t, i))\right)+\lambda_{\text {post }},
\end{aligned}
$$

where

$$
\sigma(\varphi, t, i)=\Phi_{i}, \quad t \in\left[t_{\text {post }}(i), t_{\text {post }}(i)+T_{\text {post }}(i)\right) .
$$

With regards to the values of $\omega$, we will assume that $\omega \in \operatorname{range}(f)$. It is also clear that $\varphi\left(t_{\text {post }}(i)\right)=\Phi_{i}$, $i=1,2, \ldots$ according to the definition of variable $\Phi_{i}$.

Let $t \in\left[t_{\text {post }}(i), t_{\text {post }}(i)+T_{\text {post }}(i)\right)$, then the last equation in (3.3) can be explicitly integrated giving rise to

$$
\begin{aligned}
z_{\text {post }}(t)= & \left.e^{-\alpha_{\text {post }}\left(t-t_{\text {post }}(i)\right)} z\left(t_{\text {post }}(i)\right)+\frac{k_{\text {post }}}{\alpha_{\text {post }}}\left(1-e^{-\alpha_{\text {post }}\left(t-t_{\text {post }}(i)\right)}\right) G(\sigma(\varphi, t, i))\right) \\
& +\left(\frac{\lambda_{\text {post }}}{\alpha_{\text {post }}}+I_{\text {post }}\right)\left(1-e^{-\alpha_{\text {post }}\left(t-t_{\text {post }}(i)\right)}\right)
\end{aligned}
$$

Thus taking (3.3) and (3.4) into account and approximating the function $f(\cdot)$ by a linear one ${ }^{2}, f\left(z_{\text {post }}\right)=$ $f^{*}+f_{0} z_{\text {post }}$, we can now express $\varphi(t)$ for $t \in\left[t_{\text {post }}(i), t_{\text {post }}(i)+T_{\text {post }}(i)\right)$ as:

$$
\begin{aligned}
\varphi(t)= & \varphi\left(t_{\text {post }}(i)\right)+\omega\left(t-t_{\text {post }}(i)\right)-f^{*}\left(t-t_{\text {post }}(i)\right)-f_{0}\left[\frac{1}{\alpha_{\text {post }}}\left(1-e^{-\alpha_{\text {post }}\left(t-t_{\text {post }}(i)\right)}\right) z\left(t_{\text {post }}(i)\right)\right. \\
& \left.+\left(\frac{k_{\text {post }}}{\alpha_{\text {post }}} G(\sigma(\varphi, t, i))+\left(\frac{\lambda_{\text {post }}}{\alpha_{\text {post }}}+I_{\text {post }}\right)\right)\left(\left(t-t_{\text {post }}(i)\right)-\frac{1-e^{-\alpha_{\text {post }}\left(t-t_{\text {post }}(i)\right)}}{\alpha_{\text {post }}}\right)\right] .
\end{aligned}
$$

\footnotetext{
${ }^{2}$ This is a plausible assumption given that the curve shown in Fig. 4 and depicting dependence of frequency on the excitation parameter can be approximated by a segment of a line.
} 
Noticing that $\varphi\left(t_{\text {post }}(i)+T_{\text {post }}(i)\right)=\Phi_{i+1}, \varphi\left(t_{\text {post }}(i)\right)=\Phi_{i}$, denoting $z_{i}=z_{\text {post }}\left(t_{\text {post }}(i)\right), z_{i+1}=$ $z_{\text {post }}\left(t_{\text {post }}(i)+T_{\text {post }}(i)\right)$, and using the fact that $\varphi(t)$ is continuous in $t$, we arrive at

$$
\begin{aligned}
\Phi_{i+1}= & \Phi_{i}+\omega T_{\text {post }}(i)-f^{*} T_{\text {post }}(i)-f_{0}\left[\frac{1}{\alpha_{\text {post }}}\left(1-e^{-\alpha_{\text {post }} T_{\text {post }}(i)}\right) z_{i}\right. \\
& \left.+\left(\frac{k_{\text {post }}}{\alpha_{\text {post }}} G\left(\Phi_{i}\right)+\left(\frac{\lambda_{\text {post }}}{\alpha_{\text {post }}}+I_{\text {post }}\right)\right)\left(T_{\text {post }}(i)-\frac{1-e^{-\alpha_{\text {post }} T_{\text {post }}(i)}}{\alpha_{\text {post }}}\right)\right] \\
z_{i+1}= & e^{-\alpha_{\text {post }} T_{\text {post }}(i)} z_{i}+\left(\frac{k_{\text {post }}}{\alpha_{\text {post }}} G\left(\Phi_{i}\right)+\left(\frac{\lambda_{\text {post }}}{\alpha_{\text {post }}}+I_{\text {post }}\right)\right)\left(1-e^{-\alpha_{\text {post }} T_{\text {post }}(i)}\right)
\end{aligned}
$$

Let $\Phi^{*}, z^{*}$ be an equilibrium of (3.5). Given that $G(\cdot)$ is differentiable, and neglecting dependence of $T_{\text {post }}(i)$ on $z_{\text {post }}$ if $z_{\text {post }}$ is close enough to $z^{*}$, we can linearize the dynamics of (3.5) about $\Phi^{*}, z^{*}$ :

$$
\left(\begin{array}{c}
\Phi_{i+1}-\Phi^{*} \\
z_{i+1}-z^{*}
\end{array}\right)=K_{i}\left(\begin{array}{c}
\Phi_{i}-\Phi^{*} \\
z_{i}-z^{*}
\end{array}\right), K_{i}=\left(\begin{array}{c}
k_{11}(i) k_{12}(i) \\
k_{21}(i) \\
k_{22}(i)
\end{array}\right)
$$

where

$$
\begin{aligned}
k_{11}(i) & =1-\frac{f_{0} G_{0} k_{\text {post }}}{\alpha_{\text {post }}}\left(T_{\text {post }}(i)-\frac{1-e^{-\alpha_{\text {post }} T_{\text {post }}(i)}}{\alpha_{\text {post }}}\right) \\
k_{12}(i) & =-\frac{f_{0}}{\alpha_{\text {post }}}\left(1-e^{-\alpha_{\text {post }} T_{\text {post }}(i)}\right) \\
k_{21}(i) & =\frac{G_{0} k_{\text {post }}}{\alpha_{\text {post }}}\left(1-e^{-\alpha_{\text {post }} T_{\text {post }}(i)}\right) \\
k_{22}(i) & =e^{-\alpha_{\text {post }} T_{\text {post }}(i)} \\
G_{0} & =G^{\prime}\left(\Phi^{*}, z^{*}\right) .
\end{aligned}
$$

Denoting $\delta \Phi=\Phi^{*}-\Phi_{c}$ we rewrite $(3.6)$ as

$$
\left(\begin{array}{c}
\Phi_{i+1}-\Phi_{c} \\
z_{i+1}-z^{*}
\end{array}\right)=K_{i}\left(\begin{array}{c}
\Phi_{i}-\Phi_{c} \\
z_{i}-z^{*}
\end{array}\right)-u_{i}, u_{i}=\left(\begin{array}{c}
k_{11}(i)-1 \\
k_{21}(i)
\end{array}\right) \delta \Phi
$$

where, according to the second equation in (3.5) at $z_{i+1}=z_{i}=z^{*}$, the value of $\delta \Phi$ is

$$
\delta \Phi=G^{-1}\left(\frac{-\lambda_{\text {post }}-\alpha_{\text {post }}\left(I_{\text {post }}-z^{*}\right)}{k_{\text {post }}}\right) .
$$

Let $\sigma_{1}, \sigma_{2}$ be eigenvalues of the matrix

$$
K_{i}=\left(\begin{array}{ll}
k_{11}(i) & k_{12}(i) \\
k_{21}(i) & k_{22}(i)
\end{array}\right)
$$

Then, provided that the fluctuations of $T_{\text {post }}(i)$ are sufficiently small, condition $\left|\sigma_{1}\right|<1,\left|\sigma_{2}\right|<1$ ensures that the fixed point $\Phi^{*}, z^{*}$ is stable in the sense of Lyapunov.

The eigenvalues of $K_{i}$ can be expressed as

$$
\sigma_{1,2}=\frac{\left(k_{11}(i)+k_{22}(i)\right) \pm \sqrt{\left(k_{11}(i)+k_{22}(i)\right)^{2}-4\left(k_{11}(i) k_{22}(i)-k_{12}(i) k_{21}(i)\right)}}{2} .
$$

According to (3.7), (3.9), checking if $\left|\sigma_{1}\right|<1,\left|\sigma_{2}\right|<1$ holds requires availability of the estimates/values of $f_{0}$ and $G_{0}$. The value of $f_{0}$ can be explicitly inferred from Fig. $4: f_{0} \simeq 0.025$, and the value of $G_{0}$ belongs to $[0,2 \pi]$. Figure 5 shows plots of $\left|\sigma_{1}\right|,\left|\sigma_{2}\right|$ as functions of $k_{\text {post }}$ for $\alpha_{\text {post }}=0.01, G_{0}=2 \pi$, and two different values of $T_{\text {post }}: T_{\text {post }}=35$ (black curve), and $T_{\text {post }}=50$ (grey curve). According to the figure, there is a range of values of $k_{\text {post }}$ for which both $\left|\sigma_{1}\right|$ and $\left|\sigma_{2}\right|$ are less than one, and hence the fixed point is stable.

Summarizing the analysis above one can conclude that 


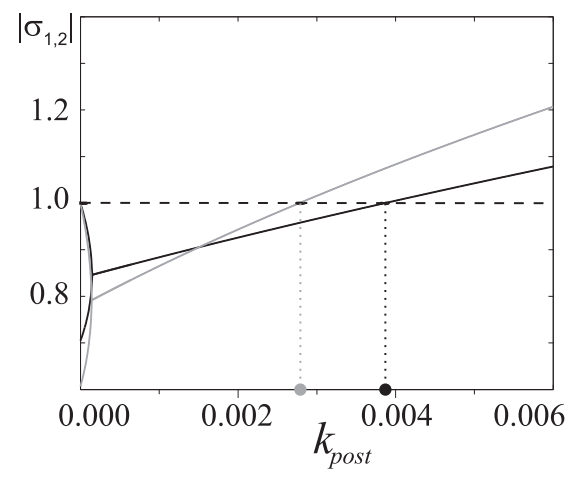

FiguRE 5. Stability diagram derived from the local analysis of the fixed points of (3.5). Grey line shows the values of $\left|\sigma_{1}\right|,\left|\sigma_{2}\right|$ (eigenvalues of the Jacobian of (3.5), see also (3.9)) as functions of $k_{\text {post }}$ for $T_{\text {post }}=50$. Black line depicts the values of $\left|\sigma_{1}\right|,\left|\sigma_{2}\right|$ for $T_{\text {post }}=35$. Other parameter values were set as follows: $\alpha_{\text {post }}=0.01, f_{0}=0.025$, $G_{0}=2 \pi, g_{\text {syn }}=0$. Grey and black circles indicate critical values of $k_{\text {post }}^{*}\left(T_{\text {post }}\right)$, for $T_{\text {post }}=50$ and $T_{\text {post }}=35$ respectively, at which the fixed point $\Phi^{*}, z^{*}$ becomes unstable.

- if $f_{0}, G_{0}>0$ then, for a broad range of $T_{\text {post }}$, there will always exist values of the STDP parameters, $k_{\text {post }}, \alpha_{\text {post }}$, such that the fixed point of (3.5) is locally exponentially stable for these values;

- the values of relative phase mismatches, $\delta \Phi$, corresponding to the fixed point depends reciprocally on the value of $k_{\text {post }}$; the larger is the absolute value of $k_{\text {post }}$, the smaller is the value of $|\delta \Phi|$

- however, if the values of $k_{\text {post }}$ are made large enough, i.e. when $\max \left\{\left|\sigma_{1}\right|,\left|\sigma_{2}\right|\right\}>1$ (which is always possible to achieve, see (3.7), (3.9)), the corresponding fixed point becomes unstable.

Remark 3.1. An alternative strategy for assessing stability of the equilibria of (3.3) can be carried out without explicit integration of the second equation in (3.3). If $k_{\text {post }} \in \mathbb{R}_{>0}, \alpha_{\text {post }} \in \mathbb{R}_{>0}$ are sufficiently small then solutions of (3.3) can be approximated by that of

$$
\begin{aligned}
\dot{\varphi} & =\omega-f\left(z_{\text {post }}\right) \\
\dot{z}_{\text {post }} & \left.=\alpha_{\text {post }}\left(I_{\text {post }}-z_{\text {post }}\right)+k_{\text {post }} G\left(\varphi-\Phi_{c}\right)\right)+\lambda_{\text {post }} .
\end{aligned}
$$

It is clear that equilibria of (3.10) can be determined from

$$
\begin{aligned}
z^{*} & =f^{-1}(\omega) \\
\Phi^{*} & \in G^{-1}\left(\frac{-\alpha_{\text {post }}\left(I_{\text {post }}-f^{-1}(\omega)\right)-\lambda_{\text {post }}}{k_{\text {post }}}\right)+\Phi_{c}
\end{aligned}
$$

where $G^{-1}$ is, in general, a set-valued function. If $\Phi^{*}$ is such that $\partial G / \partial \varphi\left(\Phi^{*}-\Phi_{c}\right)>0$ then one can conclude that $\Phi^{*}, z^{*}$ is an asymptotically stable equilibrium of (3.3). The conclusion follows from the analysis of the time-derivative of the following Lyapunov candidate:

$$
V\left(\varphi, z_{\text {post }}\right)=\int_{z^{*}}^{z_{\text {post }}}\left(f(s)-f\left(z^{*}\right)\right) d s+k_{\text {post }} \int_{\Phi^{*}}^{\varphi} G(s)-G\left(\Phi^{*}\right) d s
$$

followed by invoking the Barbalatt's lemma for demonstrating asymptotic convergence of $\varphi(t)$ to $\Phi^{*}$.

In order to proceed with the analysis further a technical result provided in the next paragraphs is required 


\subsection{Local non-uniform small gain theorem}

Consider a system with input, $u$, and let the evolution of its state, $x$, be governed by

$$
\dot{x}=f(x, u(t), t),
$$

where the function $f: \mathbb{R}^{n} \times \mathbb{R} \times \mathbb{R} \rightarrow \mathbb{R}^{n}$ is locally bounded in $x, u$, bounded in $t$, and $u: \mathbb{R} \rightarrow \mathbb{R}$ is a continuous function. For the sake of notational compactness we denote $\|z(\tau)\|_{\infty,[a, b]}=\max _{\tau \in[a, b]}\|z(\tau)\|$, where $z$ is a function defined on $[a, b]$. Let $\Omega\left(t_{0}, t\right)=\Omega_{x} \times \Omega_{u}\left(t_{0}, t\right), \Omega_{x}:\left\{x \in \mathbb{R} \mid\|x\| \leq \Delta_{x}\right\}, \Delta_{x} \in \mathbb{R}_{>0}$, $\Omega_{u}\left(t_{0}, t\right)=\left\{u \mid u: \mathbb{R} \rightarrow \mathbb{R}, u \in \mathcal{C}^{0},\|u\|_{\infty,\left[t_{0}, t\right]} \leq \Delta_{u}\right\}$, and suppose that for any $t_{0} \in \mathbb{R}, t>t_{0}$ and all $\left(x_{0}, u\right) \in \Omega\left(t_{0}, t\right)$, the solutions of (3.12) satisfying $x\left(t_{0}\right)=x_{0}$ are defined, and the following holds

$$
\left\|x\left(t_{0}+T\right)\right\| \leq \beta(T)\left\|x\left(t_{0}\right)\right\|+c\|u(t)\|_{\infty,\left[t_{0}, t_{0}+T\right]}+\Delta_{p}, \Delta_{p} \in \mathbb{R}_{\geq 0}, T \in\left[0, t-t_{0}\right], c \in \mathbb{R}_{\geq 0},
$$

where $\beta(T)$ is a strictly monotone continuous function: $\lim _{t \rightarrow \infty} \beta(t)=0, \beta(0) \geq 1$. Let us suppose that for all $t_{0}$ input $u$ in (3.12) is evolving according to

$$
u\left(t_{0}\right) \geq u(t) \geq u\left(t_{0}\right)-\left(t-t_{0}\right) \gamma\left\|x\left(t^{\prime}\right)+D\left(t^{\prime}\right)\right\|_{\varepsilon}, t^{\prime} \in\left[t_{0}, t\right], t \geq t_{0}, \gamma \in \mathbb{R}_{>0},
$$

where $\|x\|_{\varepsilon}$ stands for $\|x\|-\varepsilon$ if $\|x\|>\varepsilon$ and 0 otherwise, and $D: \mathbb{R} \rightarrow \mathbb{R}$ is a bounded function: $\left\|D\left(t^{\prime}\right)\right\| \leq \Delta_{d}$ for all $t^{\prime}$. Then the following statement holds for interconnection (3.12), (3.13) (cf. [26], $[27])$

Theorem 3.2. Consider interconnection (3.12), (3.13), and let

$$
\varepsilon \geq \Delta_{p}\left(1+\beta(0) \frac{\kappa}{\kappa-d}\right)+\Delta_{d}
$$

Suppose that the domain

$$
\Omega_{\gamma}: 0 \leq \gamma \leq \beta^{-1}\left(\frac{d}{\kappa}\right)^{-1} \frac{\kappa-1}{\kappa} \frac{u\left(t_{0}\right)}{\beta(0)\left(\left\|x\left(t_{0}\right)\right\|+c\left|u\left(t_{0}\right)\right|(1+\kappa /(1-d))\right)+c\left|u\left(t_{0}\right)\right|}
$$

is not empty for some $d<1, \kappa>1$. Let $\Omega_{\Delta}=\left\{(x, u)|\beta(0)\|x\|+c| u\left|+\Delta_{p} \leq \Delta_{x},\right| u \mid \leq \Delta_{u}\right\}$.

Then the state $(x(t), u(t))$ of the interconnection is globally bounded if $\left(x_{0}, u\left(t_{0}\right)\right) \in \Omega_{\gamma} \cap \Omega_{\Delta}$. Furthermore, if there is a function $w: \mathbb{R}^{n} \rightarrow \mathbb{R}_{\geq 0}$ such that

$$
u(t) \leq u\left(t_{0}\right)-\left(t-t_{0}\right) w\left(x\left(t^{\prime}\right)\right), t^{\prime} \in\left[t_{0}, t\right], t \geq t_{0},
$$

then for every divergent and ordered infinite sequence $\left\{t_{i}\right\}, i=0,1, \ldots, t_{i}<t_{i+1}$, the following holds:

$$
\exists\left\{t_{i}^{\prime}\right\}, t_{i}^{\prime} \in\left[t_{i}, t_{i+1}\right]: \lim _{i \rightarrow \infty} w\left(x\left(t_{i}^{\prime}\right)\right)=0 .
$$

Proof. Boundedness. Let us introduce a strictly decreasing sequence $\left\{\sigma_{i}\right\}, i=0,1, \ldots$, such that $\sigma_{0}=1$, and $\sigma_{i}$ asymptotically converge to zero. Let $\left\{t_{i}\right\}, t_{i}, i=1, \ldots$ be an ordered infinite sequence of time instances such that:

$$
u\left(t_{i}\right)=\sigma_{i} u\left(t_{0}\right) .
$$

If the latter assumption does not hold then one can immediately conclude that $u(t)$ is bounded from below by 0 for $t \geq t_{0}$; it is also bounded from above by $u\left(t_{0}\right)$, for $t \geq t_{0}$. Hence $u(t)$ is bounded for all $t \geq t_{0}$. Moreover trajectory $x(t)$ is bounded for all $t \geq t_{0}$, and nothing remains to be proven. We wish to show that the amount of time needed to reach the set $\{(x, u) \mid u=0\}$ from the given initial condition is larger than any positive number, i.e. infinite.

Consider time differences $T_{i}=t_{i}-t_{i-1}$. It is clear that:

$$
T_{i} \max _{\tau \in\left[t_{i-1}, t_{i}\right]}\|x(\tau)+D(\tau)\|_{\varepsilon} \geq \frac{u\left(t_{0}\right)\left(\sigma_{i-1}-\sigma_{i}\right)}{\gamma} .
$$


Since $\max _{\tau \in\left[t_{i-1}, t_{i}\right]}\|x(\tau)+D(\tau)\|_{\varepsilon}=\|x(\tau)+D(\tau)\|_{\infty,\left[t_{i-1}, t_{i}\right]}-\varepsilon$ if $\|x(\tau)+D(\tau)\|_{\infty,\left[t_{i-1}, t_{i}\right]}>\varepsilon$, and $\max _{\tau \in\left[t_{i-1}, t_{i}\right]}\|x(\tau)+D(\tau)\|_{\varepsilon}=0$ overwise, we can see from (3.17) that

$$
T_{i} \geq\left\{\begin{array}{l}
\frac{u\left(t_{0}\right)\left(\sigma_{i-1}-\sigma_{i}\right)}{\gamma} \frac{1}{\|x(\tau)+D(\tau)\|_{\infty,\left[t_{i-1}, t_{i}\right]-\varepsilon}},\|x(\tau)+D(\tau)\|_{\infty,\left[t_{i-1}, t_{i}\right]}>\varepsilon \\
\infty,\|x(\tau)+D(\tau)\|_{\infty,\left[t_{i-1}, t_{i}\right] \leq \varepsilon}
\end{array}\right.
$$

We will consider the case when $\|x(\tau)+D(\tau)\|_{\infty,\left[t_{i-1}, t_{i}\right]}-\varepsilon>0$ for all $i=1,2, \ldots$ (since $u(t)$ is clearly bounded overwise).

In addition to $\left\{t_{i}\right\}$ we introduce another auxiliary sequence $\left\{\tau_{i}\right\}, \tau_{i}=\tau^{*}, \tau^{*} \in \mathbb{R}_{>0}, i=1, \ldots$ Given that the partial sums $\sum_{i} \tau_{i}=\sum_{i} \tau^{*}$ diverge we can conclude that proving the implication

$$
T_{i} \geq \tau^{*} \Rightarrow T_{i+1} \geq \tau^{*} \forall i
$$

will automatically assure that $x(t), u(t)$ are bounded for all $t \geq t_{0}$. We prove (3.19) by induction wrt $i$.

Let us pick

$$
\tau^{*}=\beta^{-1}(d / \kappa), d \in(0,1),
$$

and select the value of $\gamma$ such that (3.14) holds.

Let $x\left(t_{0}\right), u\left(t_{0}\right)$ be such that $\beta(0)\left\|x\left(t_{0}\right)\right\|+c\left|u\left(t_{0}\right)\right|+\Delta_{p}<\Delta_{x}, 0<u\left(t_{0}\right)<\Delta_{0}$. The function $u(t)$ is non-increasing over $\left[t_{0}, t_{i}\right]$. This ensures that $u \in \Omega_{u}\left(t_{0}, t_{i}\right)$, and hence $x\left(t_{i}\right), u\left(t_{i}\right) \in \Omega_{\Delta}$ for all $t_{i}$. Suppose that $T_{j} \geq \tau^{*}$ for $1 \leq j \leq i-1$. Therefore

$$
\begin{aligned}
\|x(\tau)\|_{\infty,\left[t_{i-t}, t_{i}\right]} & \leq \beta(0)\left\|x\left(t_{i-1}\right)\right\|+c u\left(t_{0}\right) \sigma_{i-1} \leq \beta(0)\left[\beta\left(T_{i-1}\right)\left\|x\left(t_{i-2}\right)\right\|+c u\left(t_{0}\right) \sigma_{i-2}\right]+c u\left(t_{0}\right) \sigma_{i-1} \\
& \leq \beta(0) \beta^{2}\left(\tau^{*}\right)\left\|x\left(t_{i-3}\right)\right\|+P_{2}
\end{aligned}
$$

where

$$
P_{2}=\beta(0)\left[\beta\left(\tau^{*}\right) c \sigma_{i-3}+c \sigma_{i-2}\right] u\left(t_{0}\right)+c \sigma_{i-1} u\left(t_{0}\right)+\beta(0)\left[\beta\left(\tau^{*}\right) \Delta_{p}+\Delta_{p}\right]+\Delta_{p}
$$

Repeating this iteration with respect to $i$ leads to

$$
\begin{aligned}
& \|x(\tau)\|_{\infty,\left[t_{i-t}, t_{i}\right]} \leq \beta(0) \beta^{3}\left(\tau^{*}\right)\left\|x\left(t_{i-4}\right)\right\|+P_{3} \\
& P_{3}=c u\left(t_{0}\right) \beta(0)\left[\beta^{2}\left(\tau^{*}\right) \sigma_{i-4}+\beta\left(\tau^{*}\right) \sigma_{i-3}+\sigma_{i-2}\right]+\sigma_{i-1} c u\left(t_{0}\right)+\Delta_{p} \beta(0)\left[\beta\left(\tau^{*}\right)^{2}+\beta\left(\tau^{*}\right)+1\right] \\
& +\Delta_{p}=c u\left(t_{0}\right) \beta(0)\left[\sum_{j=0}^{2} \beta^{j}\left(\tau^{*}\right) \sigma_{i-j-2}\right]+c u\left(t_{0}\right) \sigma_{i-1}+\Delta_{p} \beta(0)\left[\sum_{j=0}^{2} \beta^{j}\left(\tau^{*}\right)\right]+\Delta_{p},
\end{aligned}
$$

and after $i-1$ steps we obtain

$$
\begin{aligned}
& \|x(\tau)\|_{\infty,\left[t_{i-t}, t_{i}\right]} \leq \beta(0) \beta\left(\tau^{*}\right)^{i-1}\left\|x\left(t_{0}\right)\right\|+P_{i-1} \\
& P_{i-1}=c u\left(t_{0}\right) \beta(0)\left[\sum_{j=0}^{i-2} \beta^{j}\left(\tau^{*}\right) \sigma_{i-j-2}\right]+c u\left(t_{0}\right) \sigma_{i-1}+\Delta_{p} \beta(0)\left[\sum_{j=0}^{i-2} \beta^{j}\left(\tau^{*}\right)\right]+\Delta_{p} .
\end{aligned}
$$

Rearranging terms in (3.17) results in

$$
T_{i} \geq \frac{\sigma_{i-1}-\sigma_{i}}{\sigma_{i-1}} \frac{u\left(t_{0}\right)}{\gamma} \frac{1}{\sigma_{i-1}^{-1}\left(\|x(\tau)+D(\tau)\|_{\infty,\left[t_{i-1}, t_{i}\right]}-\varepsilon\right)} .
$$

Hence, if we can show that there exist an $x_{0}$ such that such that for some $\Delta_{0} \in \mathbb{R}_{\geq 0}$ :

$$
\frac{\sigma_{i-1}-\sigma_{i}}{\sigma_{i-1}} \frac{u\left(t_{0}\right)}{\tau^{*}} \geq \Delta_{0}
$$


the following holds

$$
\gamma \sigma_{i-1}^{-1}\left(\|x(\tau)+D(\tau)\|_{\infty,\left[t_{i-1}, t_{i}\right]}-\varepsilon\right) \leq \gamma B\left(x_{0}\right) \leq \Delta_{0} \forall i
$$

where $B(\cdot)$ is a function of $x_{0}$, then implication (3.19) will obviously follow.

Consider $\sigma_{i-1}^{-1}\left(\|x(\tau)+D(\tau)\|_{\infty,\left[t_{i-1}, t_{i}\right]}-\varepsilon\right)$, and let

$$
\sigma_{i}=\frac{1}{\kappa^{i}}, \kappa>1
$$

According to (3.21) we have:

$$
\begin{aligned}
& \sigma_{i-1}^{-1}\left(\|x(\tau)+D(\tau)\|_{\infty,\left[t_{i-1}, t_{i}\right]}-\varepsilon\right) \leq \beta(0)\left[\sigma_{i-1}^{-1} \beta\left(\tau^{*}\right)^{i-1}\right]\left\|x\left(t_{0}\right)\right\|+\sigma_{i-1}^{-1} P_{i-1}+\sigma_{i-1}^{-1} \Delta_{d} \\
& =\beta(0)\left(\kappa \beta\left(\tau^{*}\right)\right)^{i-1}\left\|x\left(t_{0}\right)\right\|+\kappa^{i-1}\left(P_{i-1}+\Delta_{d}\right) \\
& =\beta(0)\left(\kappa \beta\left(\tau^{*}\right)\right)^{i-1}\left\|x\left(t_{0}\right)\right\|+c u\left(t_{0}\right) \beta(0) \kappa\left[\sum_{j=0}^{i-2} \beta^{j}\left(\tau^{*}\right) \kappa^{j}\right]+c u\left(t_{0}\right)+ \\
& \kappa^{i-1}\left[\Delta_{p}\left(\beta(0) \sum_{j=0}^{i-2} \beta^{j}\left(\tau^{*}\right)+1\right)+\Delta_{d}-\varepsilon\right] .
\end{aligned}
$$

Hence choosing the value of $\tau^{*}$ as in (3.20)

$$
\kappa \beta\left(\tau^{*}\right)=d, d \in(0,1)
$$

letting $\varepsilon \geq \Delta_{p}\left(1+\beta(0) \frac{\kappa}{\kappa-d}\right)+\Delta_{d}$, and noticing that

$$
\kappa^{i-1}\left[\Delta_{p}\left(\beta(0) \sum_{j=0}^{i-2} \beta^{j}\left(\tau^{*}\right)+1\right)+\Delta_{d}-\varepsilon\right]=k^{i-1}\left[\Delta_{p}\left(\frac{\beta(0)}{1-d / k}+1\right)+\Delta_{d}-\varepsilon\right],
$$

we can conclude that

$$
\sigma_{i-1}^{-1}\left(\|x(\tau)+D(\tau)\|_{\infty,\left[t_{i-1}, t_{i}\right]}-\varepsilon\right) \leq \beta(0)\left\|x\left(t_{0}\right)\right\|+c u\left(t_{0}\right)\left(1+\frac{\beta(0) \kappa}{1-d}\right)=B\left(x_{0}\right) .
$$

Solving (3.20), (3.22) for respect to $\Delta_{0}$ results in

$$
\Delta_{0}=\frac{\kappa-1}{\kappa}\left[\beta^{-1}\left(\frac{d}{\kappa}\right)\right]^{-1} u\left(t_{0}\right) .
$$

This in turn implies that for all $\left(x\left(t_{0}\right), u\left(t_{0}\right)\right) \in \Omega_{\Delta}$ which, in addition, satisfy:

$$
\gamma \leq \frac{\kappa-1}{\kappa}\left[\beta^{-1}\left(\frac{d}{\kappa}\right)\right]^{-1} \frac{u\left(t_{0}\right)}{\beta(0)\left\|x\left(t_{0}\right)\right\|+c\left|u\left(t_{0}\right)\right|\left(1+\frac{\kappa \beta(0)}{1-d}\right)}
$$

the following implication must hold: $T_{i} \geq \tau^{*} \Rightarrow T_{i+1} \geq \tau^{*}$. Therefore, trajectories $x(t), u(t)$ passing through $x\left(t_{0}\right), u\left(t_{0}\right)$ at $t=t_{0}$ are bounded in forward time.

Convergence. Let us show that (3.15) implies (3.16). Suppose that this is not the case, and for an ordered diverging sequence $\left\{t_{i}\right\}$ there is a $\delta>0$ such that $w\left(x\left(t^{\prime}\right)\right)>\delta, \forall t^{\prime} \in\left[t_{i-1}, t_{i}\right], i=1,2, \ldots$ Hence

$$
u\left(t_{i}\right) \leq u\left(t_{i-1}\right)-\left(t_{i}-t_{i-1}\right) \delta \leq u\left(t_{0}\right)-\left(t_{0}-t_{i-1}\right) \delta .
$$

According to the first part of the proposition $u\left(t_{i}\right)$ is bounded for all $i$. On the other hand, using (3.23) one can conclude that for any given arbitrarily large $M$, there is an $n>0$ such that $u\left(t_{n}\right) \leq-M$. Thus we have reached contradiction which proves (3.16). 


\subsection{Phase adaptation}

So far we have established that STDP mechanisms (2.1), (2.2) may result in the emergence of the stable fixed point of the relative phase dynamics. The relative phase values corresponding to this fixed point, however, do not coincide with the reference, $\Phi_{c}$. Even though there is a room for optimizing the phase mismatch, $\delta \Phi$, by increasing the values of STDP gains, discrete nature of the regulatory feedback, imposes a limitation on the degree to which such an optimization can be used without incurring penalties in the form of the loss of stability of the fixed point. Note that, according to (3.11), if parameters of the STDP law are chosen such that $\alpha_{\text {post }}\left(f^{-1}(\omega)-I_{\text {post }}\right)-\lambda_{\text {post }}(t)=0$ then the relative phase variable, $\Phi$, (in a neighborhood of the locking state) locks to the the reference $\Phi_{c}$ asymptotically. The problem is, however that the value of natural frequencies mismatch, $\omega$, is unknown a-priori. Thus annihilating the error by choosing the values of $\alpha_{\text {post }}, I_{\text {post }}$ (or $\alpha_{\text {pre }}, I_{\text {pre }}$ for the presynaptic feedback) is not a viable option. This motivates introduction of an extra dynamical process in the system that is capable of producing such an adjustment.

An important consequence of the stability analysis above, specifically (3.3) - (3.8), is that if $\lambda_{\text {post }}$ is allowed to vary then, subject to the choice of $k_{\text {post }}, \alpha_{\text {post }}$, the dynamics of $(2.8)$ in which $z_{\text {post }}$ evolves in accordance with (2.2) locally satisfies the following constraint:

$$
\begin{aligned}
\left\|\begin{array}{c}
\varphi(t)-\Phi_{c} \\
z_{\text {post }}(t)-z^{*}
\end{array}\right\| & \leq \beta\left(t-t_{0}\right)\left\|\begin{array}{c}
\varphi\left(t_{0}\right)-\Phi_{c} \\
z_{\text {post }}\left(t_{0}\right)-z^{*}
\end{array}\right\|+c \cdot \max _{t \in\left[t_{0}, t\right]}\left\|\lambda^{*}-\lambda_{\text {post }}(t)\right\|, t \geq t_{0} \\
\lambda^{*} & =-\alpha_{\text {post }}\left(I_{\text {post }}-f^{-1}(\omega)\right),
\end{aligned}
$$

where $\beta(\cdot)$ is a strictly monotone, positive, and non-increasing function vanishing asymptotically at infinity, and $c$ is a non-negative constant.

As before we will suppose that only one oscillator (postsynaptic) is equipped with the STDP mechanism, i.e. only $\lambda_{\text {post }}$ is adapting. Consider system (3.3) in which the function $\lambda_{\text {post }}(t)$ evolves according to the following simple rule:

$$
\lambda_{\text {post }}\left(t_{0}\right)+\Gamma \int_{t_{0}}^{t}\left|\sigma(\varphi, \tau, i)-\Phi_{c}\right| d t \leq \lambda_{\text {post }}(t) \leq \lambda_{\text {post }}\left(t_{0}\right)+\gamma \int_{t_{0}}^{t}\left|\sigma(\varphi, \tau, i)-\Phi_{c}\right| d t, \Gamma, \gamma \in \mathbb{R}_{>0},
$$

where $\sigma(\varphi, t, i)=\Phi_{i}$ for all $t \in\left[t_{\text {post }}(i), t_{\text {post }}(i)+T_{\text {post }}(i)\right), T_{\text {post }}(i)>0$, and $\left|\lambda^{*}-\lambda_{\text {post }}\left(t_{0}\right)\right| \leq M_{\lambda}$. The value of $M_{\lambda}$ is supposed to be small enough so that there is a neighborhood of $\Phi_{c}$ : $\left|\varphi-\Phi_{c}\right| \leq M_{\Phi}$ such that (3.24) holds for all

$$
\left|\varphi(t)-\Phi_{c}\right| \leq M_{\Phi},\left|\lambda^{*}-\lambda_{\text {post }}(t)\right| \leq M_{\lambda}
$$

According to Theorem 3.2, if at any given $t_{0}$ variables $\varphi\left(t_{0}\right), \lambda_{\text {post }}\left(t_{0}\right)$ of the combined system (3.24), (3.25) satisfy

$$
\left|\varphi\left(t_{0}\right)-\Phi_{c}\right| \leq M_{\Phi}, 0 \leq \lambda^{*}-\lambda_{\text {post }}\left(t_{0}\right) \leq M_{\lambda}
$$

then picking

$$
0 \leq \gamma \leq \beta^{-1}\left(\frac{d}{\kappa}\right)^{-1} \frac{\kappa-1}{\kappa} \frac{M_{\lambda}}{\beta(0)\left(M_{\Phi}+c|u|(1+\kappa /(1-d))\right)+c M_{\lambda}}
$$

ensures that $\varphi(t)$ converges to $\Phi_{c}$ asymptotically.

Consider a modified version of (3.25):

$$
\lambda_{\text {post }}(t)=\lambda_{\min }+\frac{\lambda_{\max }-\lambda_{\min }}{2}\left(1-\sin \left(\frac{2}{\lambda_{\max }-\lambda_{\min }}\left(\lambda_{0}+\gamma \int_{t_{0}}^{t}\left|\sigma(\varphi, \tau, i)-\Phi_{c}\right| d \tau\right)\right)\right),
$$

where the value of $\gamma$ is chosen according to (3.27), and $\lambda^{*} \in\left[\lambda_{\min }, \lambda_{\max }\right]$. Suppose that for any continuous $\lambda_{\text {post }}(t): \lambda_{\min } \leq \lambda_{\text {post }}(t) \leq \lambda_{\max }$

1) solutions of (3.3) are defined; 
2) for each $\lambda_{\text {post }}$ fixed, $\lambda_{\text {post }} \in\left[\lambda_{\min }, \lambda_{\max }\right]$, there is a unique attractor, which is locally exponentially stable;

3) there is a neighborhood of $\Phi_{c}:\left|\varphi-\Phi_{c}\right|<M_{\Phi}$ such that (3.24) holds.

It is therefore clear that one can pick $\gamma$ so small that solutions of the combined system (3.3), (3.28) will eventually converge into the domain specified by (3.26). In this domain the function $\lambda_{\text {post }}$ defined in (3.28) will satisfy (3.25), albeit possibly with different constants $\gamma, \Gamma$. Hence, Theorem 3.2 applies, and thus one can pick $\gamma>0$ sufficiently small so that $\lim _{t \rightarrow \infty, i \rightarrow \infty} \sigma(\varphi, t, i)=\Phi_{c}$. This, in turn implies that $\varphi(t) \rightarrow \Phi_{c}$ at $t \rightarrow \infty$.

This adaptation mechanism described above is in essence a slow fluctuation of the excitation thresholds. The frequency of these fluctuations increases if absolute values of relative phase are far away from the desired ones. The frequency slows down when relative phase approaches its desired value, i.e. the reference $\Phi_{c}$.

\section{Numerical study of the relative phase dynamics in coupled neural oscillators with STDP-regulated parameters}

So far we have established analytically that phase-locking of the relative phase dynamics may occur in a system of generic neural oscillators equipped with an STDP mechanism affecting parameters regulating natural frequencies of oscillations in the system. We have also shown that without an adaptation mechanisms adjusting parameters of the STDP law (or, alternatively, parameters of the oscillators) precise phase locking to a given relative phase value may not be possible to achieve. However, if an adaptation mechanism is added (e.g. (3.28)) then the values of relative phase could be stirred to small neighborhoods of the reference.

These results, however, are local and their validity is largely confined to the domain in which approximation (3.1) holds. Second, as is often the case in the models of considered type, variables $z_{\text {pre }}, z_{\text {post }}$ could be viewed as bifurcation parameters of the model, and as such they are likely to affect dynamics of the interconnection beyond cases captured in the local analysis above. Therefore, in order to ensure that our earlier conclusions retain plausibility, further and extensive numerical studies are required. These will be presented in the next paragraphs.

\subsection{Spiking phase map}

In order to characterize and analyze post- to presynaptic timing in (2.8), including cases when $z_{\text {pre }}$ and $z_{\text {post }}$ are varying with time, we introduce spiking phase map [16]. The map itself is constructed as follows. First, we define the relative spiking phase, $\Phi$, as:

$$
\Phi=\frac{t_{\text {post }}-t_{\text {pre }}}{T_{\text {pre }}}, \ldots 0<\Phi<1
$$

where $t_{\text {pre }}$ is the time corresponding to occurrence of a presynaptic spike, and $t_{\text {post }}$ is the time of the first postsynaptic spike generated in response to the presynaptic one; $T_{p r e}$ is the period of oscillations in the presynaptic neuron. Variable $\Phi$, therefore, may be viewed as a sample of relative phase of the oscillators that is measured at the moments of time when the post-synaptic oscillator fires. Second, having defined a sequence of $\Phi$ over time, we determine the spiking phase map as follows:

$$
\begin{aligned}
& T: \Phi_{i} \rightarrow \Phi_{i+1}, \quad i=1,2, \ldots, \\
& \Phi_{i}=\frac{t_{\text {post }}(i)-t_{\text {pre }}(i)}{T_{\text {pre }}}
\end{aligned}
$$

where $i$ is the index of transmitted spikes in the sequence. It was shown in [16] that in the case of constant $z_{\text {pre }}, z_{\text {post }}$ transformation (4.2) may be modeled by a one-dimensional point map, $\Phi_{i+1}=T\left(\Phi_{i}\right)$, where $T$ is a piece-wise continuous function on the interval $0<\Phi \leq 1$. Stable fixed points of this map correspond to the spike synchronization mode $1: 1$. Spiking phase in this mode is locked to the value of 

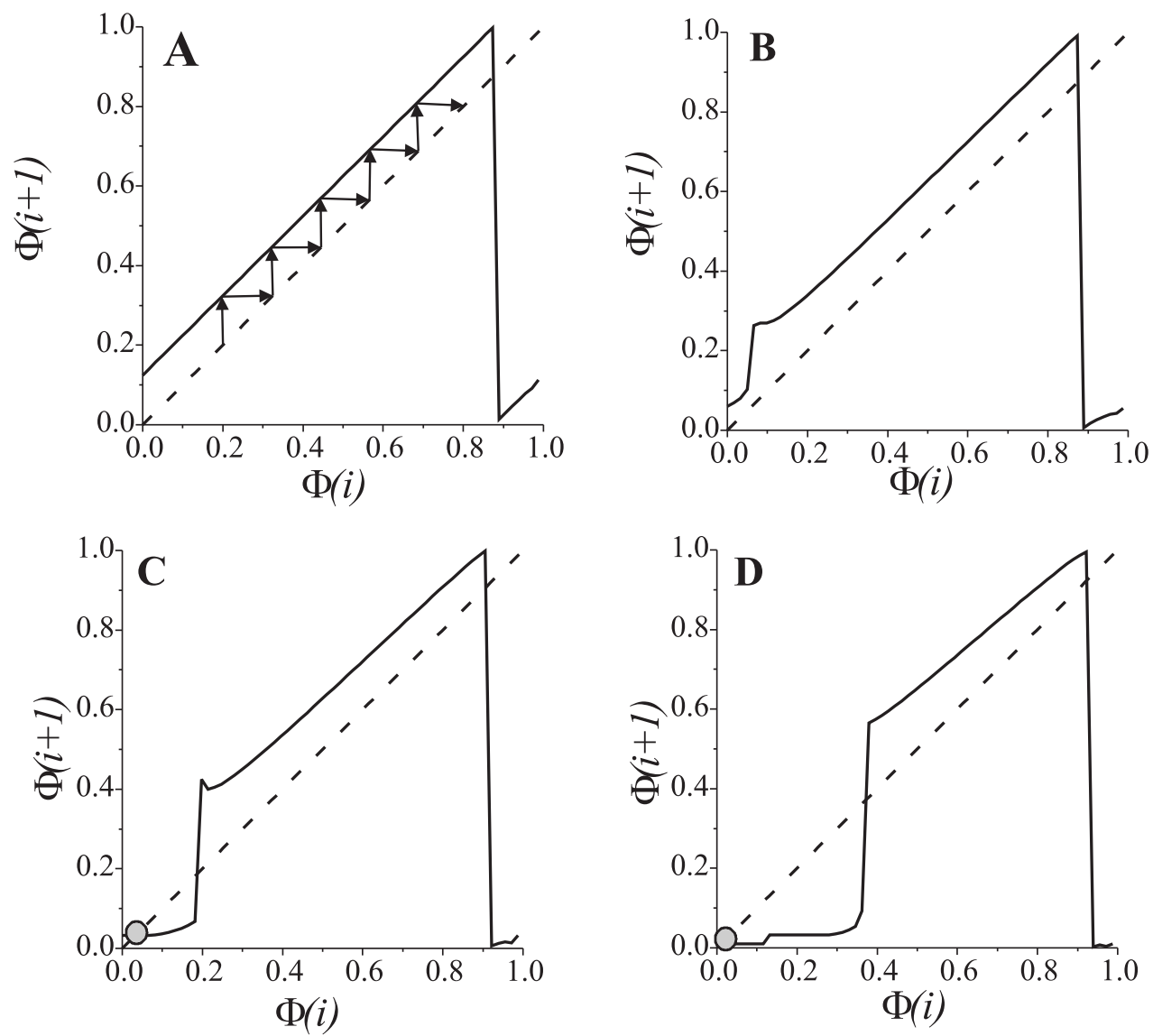

FiguRe 6. PRC curves for different coupling strengths, $\Delta I=-0.05$. A: $g_{\text {syn }}=0$. Relative phase shift is monotonically increasing as it is shown by arrows. The increase is linearly proportional to the frequency mismatch, $\Delta I$. B: PRC for small value of the synaptic coupling, $g_{\text {syn }}=0.008$. Monotonically increasing phase is pulled towards the abscissa in the vicinity of the origin. C: Synchronization for $g_{\text {syn }}=0.04$. Stable fixed point emerging from the tangent $(+1)$ bifurcation defines the value of the phase locked with a small synaptic transmission delay. D: Synchronization for the increased coupling strength, $g_{\text {syn }}=0.1$. The fixed point is close to zero.

the fixed point. Note, that the spiking phase map can be also viewed as a discrete version of the pulse coupled equations. These are typically used in the literature on the analysis of weakly coupled neuronal oscillators for describing dynamics of relative phases in the system. The function $T(\Phi)$ in this context is often referred to as the phase response curve (PRC). The advantage of using discrete spiking phase map instead of its continuous-time counterpart is that the discrete map, (4.2), is defined for any values of coupling strengths, provided that both systems oscillate.

Figure 6 shows typical shapes of the PRCs for (2.8). In the absence of coupling relative phase shifts increase in a monotone fashion (Fig. 6A). Adding a small coupling alternates the dynamics and, respectively, PRCs. Figure 6 B shows the spiking phase map near the tangent or +1 bifurcation. There appears to be a region (a ghost) in the figure which is pulling and trapping, for quite a long period of time, the values of $\Phi_{i}$. The effect is illustrated in more detail in Fig. 7A. Notice that the system's state may remain in a neighborhood of the synchronous mode for a rather long time. In the phase space of Eqs. (2.8) this corresponds to solutions near periodic or quasi-periodic orbits on the invariant torus. Further increase 

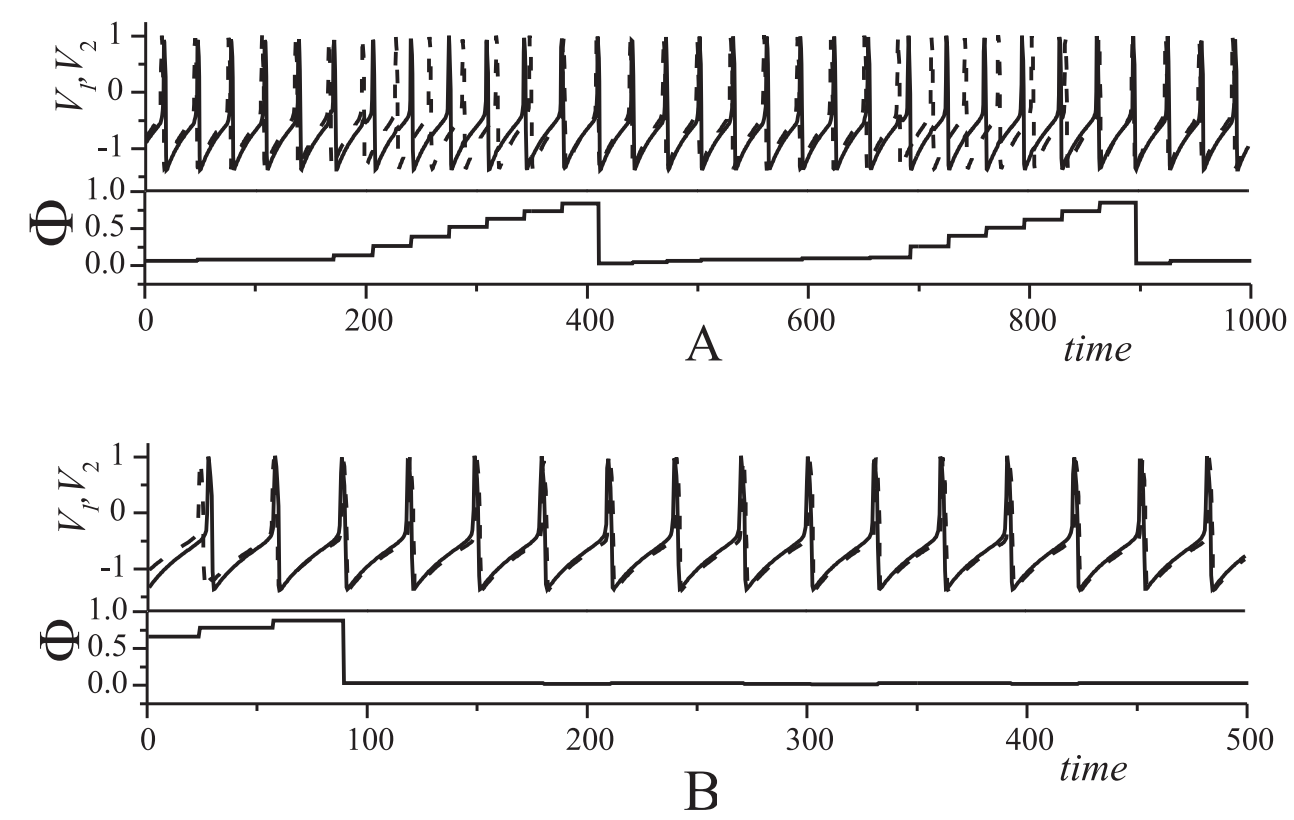

Figure 7. Oscillations in synaptically coupled oscillators Eqs. (2.8). Upper panel shows the membrane potentials in presynaptic (dashed curve) and in postsynaptic neurons (solid curve), respectively. The lower panel shows time evolution of the relative spiking phase. A: Phase pulling effect. Long lasting quasi-synchronous signals are alternating with phase reset intervals. Parameter values: $g_{\text {syn }}=0.008, \Delta I=-0.05$. B: Synchronization and phase locking due to the excitatory synaptic coupling. Parameter values: $g_{\text {syn }}=0.1, \Delta I=-0.05$.

of $g_{\text {syn }}$ leads to appearance of a stable fixed point. The fixed point corresponds to nearly synchronous firing (i.e. with almost zero phase lags) of pre and post- synaptic oscillators (Fig. 6C,D). An example of such a solution of (2.8) is shown in Fig. 7B.

\subsection{STDP with postsynaptic feedback}

First, we consider the postsynaptic STDP feedback - the one in which timing of pre- and post- synaptic events changes excitability of the postsynaptic neuron (Fig. 2A). In this case dynamics of the presynaptic neuron is not affected. Hence it is plausible to assume that the presynaptic neuron generates a sequence of spikes with a fixed, albeit unknown, frequency.

Let us investigate dynamics of relative phase for this system. As before, we approach the task by constructing and analyzing the corresponding spiking phase map. Given that the value of $T_{\text {pre }}$ is constant, the map is described as follows:

$$
T_{\text {post }}:\left\{\begin{array}{l}
\Phi_{i} \rightarrow \Phi_{i+1} \\
z_{\text {post }}\left(t_{i}\right) \rightarrow z_{\text {post }}\left(t_{i+1}\right)
\end{array} i=1,2, \ldots\right.
$$

Yet, for the sake of convenience of illustration we will only present its one-dimensional projections on the relative phase coordinate, $\Phi$.

Figure 8 illustrates spiking phase map for postsynaptic STDP driven by the pair of Hodgkin-Huxley neurons. (2.5). When the gain, $k_{\text {post }}$, increases the map undergoes through two major bifurcations. These bifurcations delimit the boundaries of STDP driven synchronization. For lower values of $k_{\text {post }}>0$ saddle-node bifurcation takes place (from Fig. 8 A to B) and stable fixed point appears. It exists until period-doubling bifurcation for relatively high $k_{\text {post }}($ Fig. $8 \mathrm{D}$ ). 

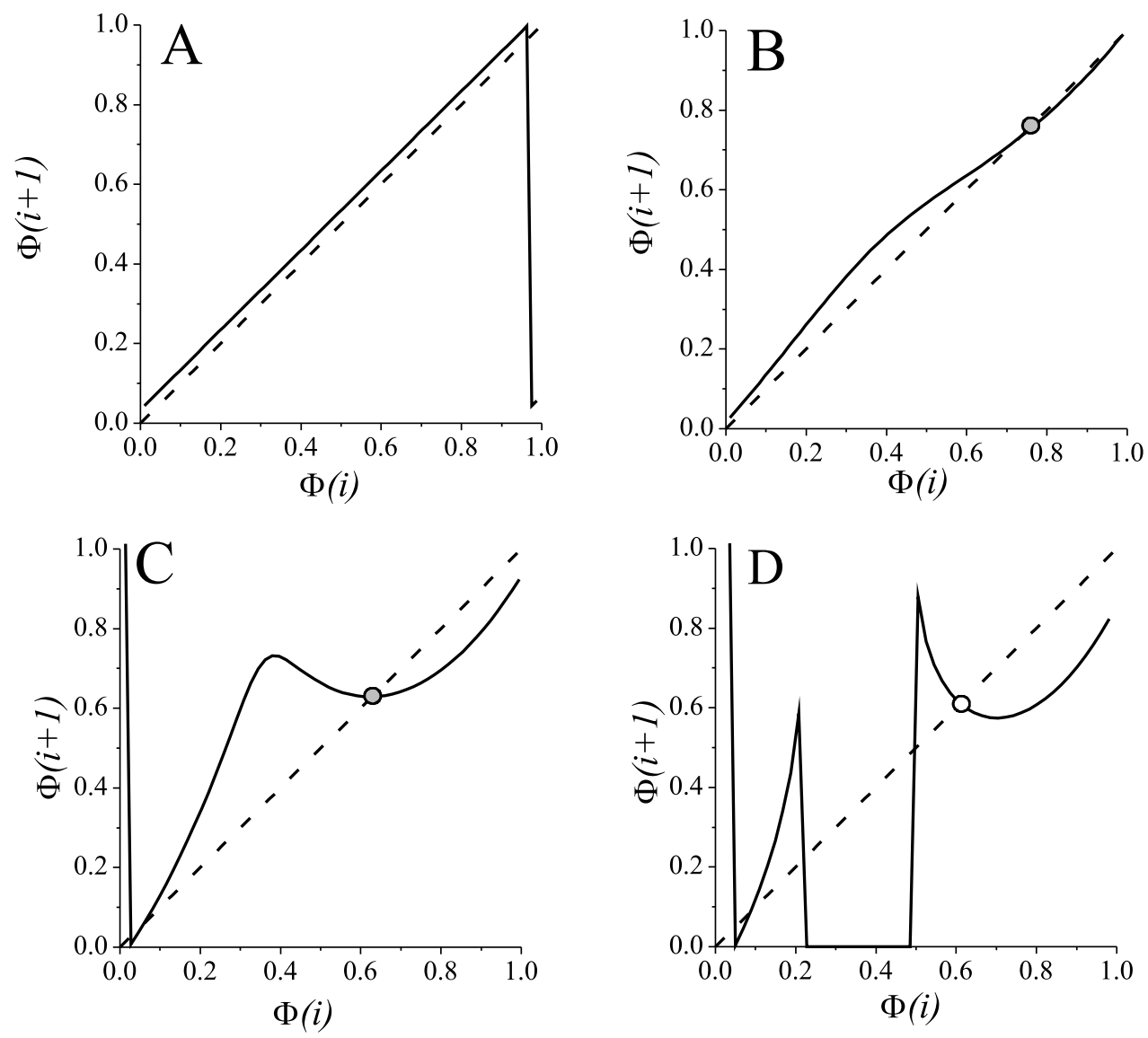

FiguRE 8. Postsynaptic STDP driven PRC curves for different feedback gains, $\Delta I=$ $-1 \mu \mathrm{A} / \mathrm{cm}^{2}, g_{\text {syn }}=0, \Phi_{c}=0.6$. A: $k_{\text {post }}=0$. Relative phase shift is monotonically increasing proportionally to the frequency mismatch, $\Delta I$. B: PRC for small value of the feedback gain, $k_{\text {post }}=0.1$. The stable fixed point corresponds to synchronization with phase shift close to the phase $\Phi_{C}$. C: PRC for $k_{\text {post }}=0.3$. D: PRC for $k_{\text {post }}=0.5$. The fixed point loses stability through the period-doubling bifurcation.

Similar scenario can be found for the reduced model (2.8). We analyze in details the period-doubling transition (Fig. 9). The STDP feedback stabilizes relative phase in a neighborhood of the reference value Corresponding PRCs are shown in Fig. 9A. The figure suggests presence of a stable fixed point, $\Phi^{*}$. If one increases the value of $k_{\text {post }}$ the fixed point $\Phi^{*}$ looses stability through the period doubling bifurcation. To the right of this critical point behavior of the system resembles a route to chaos through the period doubling cascade (Fig. 9B) [6]. Note that the value of relative phase remains in a vicinity of the fixed point even if the fixed point itself becomes unstable. The values of relative phase, however, appear to be attracting to a stable $2^{m}$-periodic orbit or to a set with a structure of a chaotic attractor. Corresponding plots of the evolution of $\Phi$ and $z_{\text {post }}$ are shown in Fig. 10A,B. Further increments of $k_{\text {post }}$ lead to a catastrophe of the attractor. The catastrophe occurs because the values of $z_{\text {post }}$ become so large that oscillations in the postsynaptic neuron disappear (see Fig. 4).

The fact that a set on which the values of $\Phi$ project resembles an object looking strikingly similar to a chaotic attractor suggests a rather unexpected function of the STDP mechanism considered here. The function is that such STDP-induced dynamics may offer a natural facility for encoding of information in the system. Indeed, if this set is a chaotic attractor then it comprises of infinite number of orbits with 

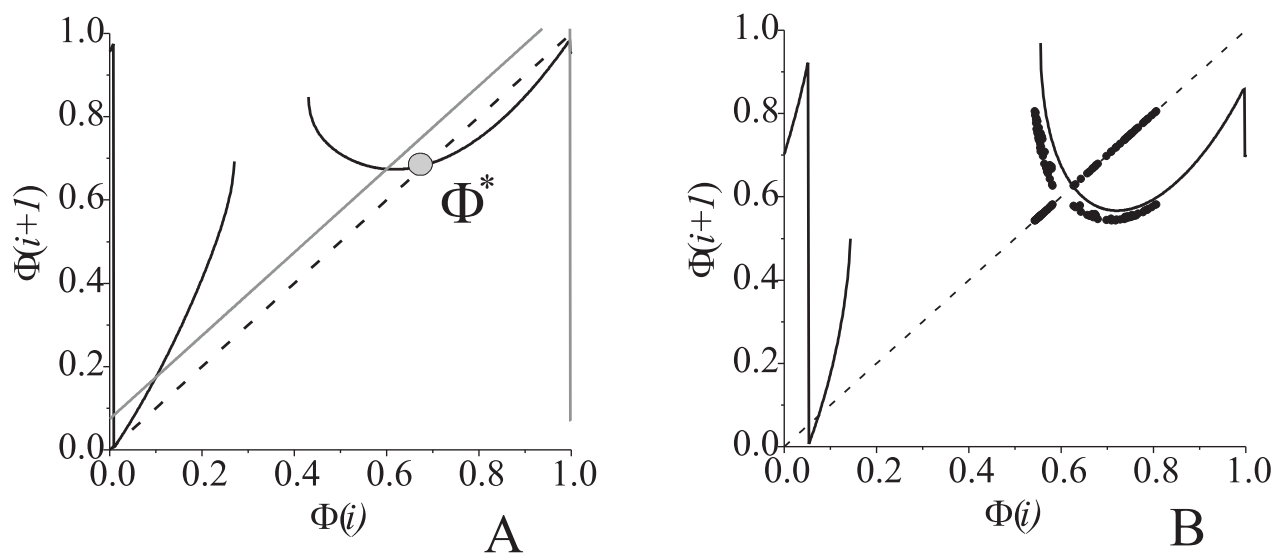

Figure 9. The PRCs for the one-dimensional approximation of the spiking phase map (4.3). A: Appearance of the stable fixed point for $k_{\text {post }}=0.002$ indicating phase locking mode in the signal transmission with reference phase $\Phi_{c}=0.6$. Grey curve shows the PRC without control. B: The PRC for large control strength, $k_{\text {post }}=0.0063$, indicating the appearance of chaotic attractor. The dots show the trajectory of the two-dimensional map (4.3). Parameter values: $\alpha_{\text {post }}=0.01, \Delta I=-0.05, g_{\text {syn }}=0$.

varying periods. Thus, in principle, a rich set of spiking sequences can be activated in such a system if an appropriate stimulus arrives.

Bifurcation diagrams characterizing dynamics of the system are shown in Fig. 11. When the values of $k_{\text {post }}$ are relatively small no locking occurs. If we increase the value of $k_{\text {post }}$ (up to the first critical point), relative phase will eventually lock to a value corresponding to nearly in-phase oscillations. In other words, STDP facilitates in-phase oscillations even if the synaptic connection is relatively week. If $k_{\text {post }}$ is increased even further (until the second critical value) relative phase locks near the reference $\Phi_{c}$. Further increments of $k_{\text {post }}$ result in gradual improvements of accuracy until, however, $k_{\text {post }}$ arrives at the third critical value. At this point the period doubling bifurcation occurs in the spiking phase map (4.3). Increasing the value of $k_{\text {post }}$ beyond this critical point gives rise to the bifurcation cascade. The latter, in turn, leads to emergence of chaotic-looking dynamics $[6,23]$ of the relative phase (Fig. 9B, Fig. 10B). This state, however, is also limited in terms of the range of admissible values of $k_{\text {post }}$. If $k_{\text {post }}$ becomes too large, i.e. it exceeds the forth critical value, oscillations in the postsynaptic neuron disappear (Fig. $11 \mathrm{~A})$.

\subsection{STDP with presynaptic feedback}

Consider system (2.8), (2.1), and (2.4). Dynamics of this configuration for $k_{\text {pre }}>0$ is illustrated in Figure 12A. One can observe that, after a relatively short transient behavior, the relative phase, $\Phi$, locks near the reference value, $\Phi_{c}$. According to the figure, the transient looks like damped oscillation relaxing asymptotically to a stable fixed point. When the relative phase locks presynaptic neuron changes its depolarization level (Fig. 12A-C, lower panel). Notice that locking occurs for both zero and nonzero synaptic coupling. Figure 12B illustrates dynamics of the system in the phase pulling mode (see Fig. $6 \mathrm{~B}$ ). If the coupling between cells is made relatively strong then presynaptic STDP feedback may destroy the in-phase synchronization mode and switch the system into the phase-locked mode determined by the value of reference phase (Fig. 12C).

The values at which relative phase locks are determined by the values of the control variable, $z_{\text {pre }}$, at the fixed point. The values of $z_{\text {pre }}$ and relative phase at the fixed point (denoted by $z_{p r e}^{*}$ and $\Phi^{*}$ 

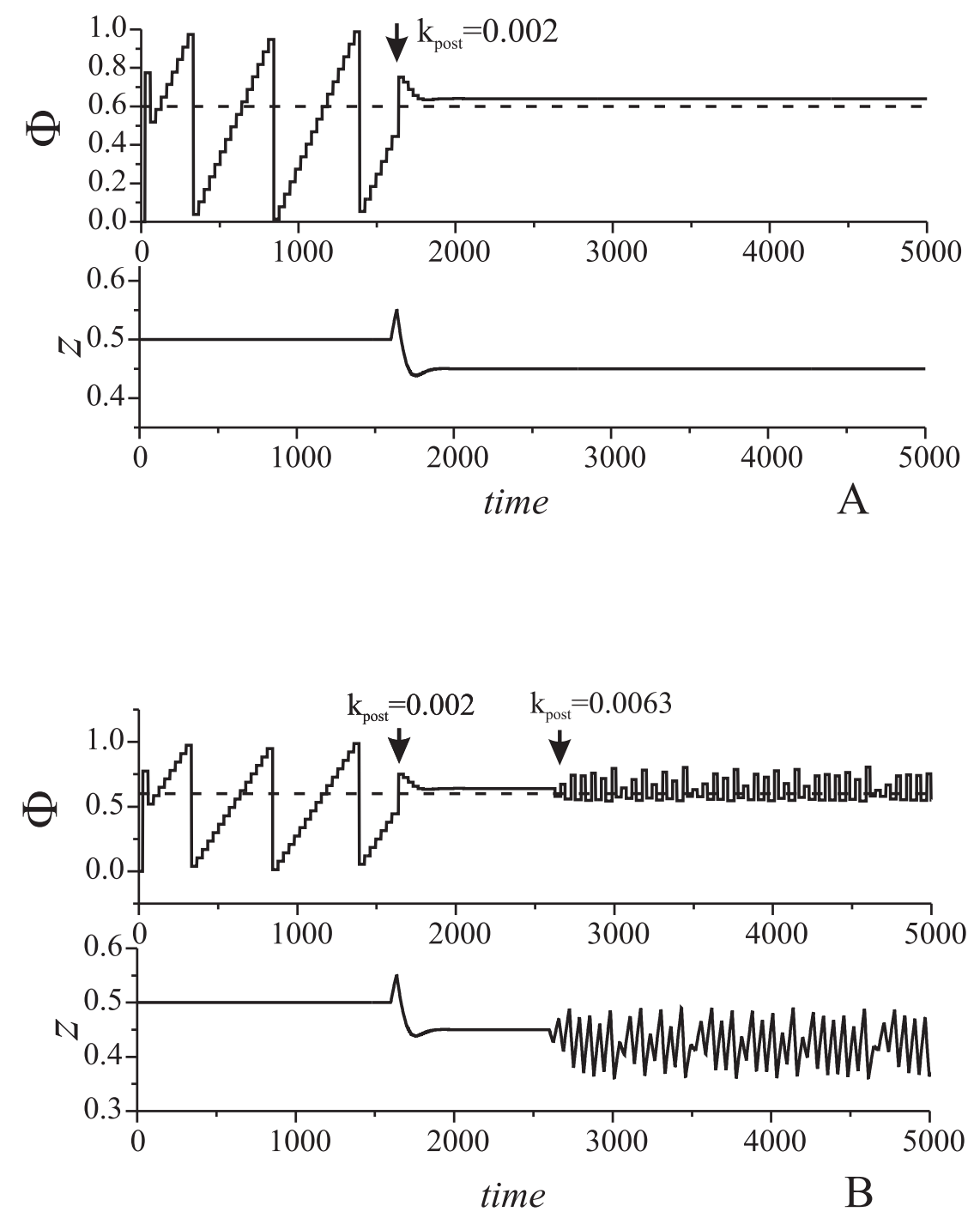

Figure 10. Evolution of spiking phase and control variable $z_{\text {post }}$ for postsynaptic control. A: Phase locking. Parameter values: $\alpha_{\text {post }}=0.01, \Delta I=-0.05, g_{\text {syn }}=0, k_{\text {post }}=$ 0.002. B: Chaotic oscillation of the spiking phase near the reference phase. The strength of the feedback is changed in two steps marked by the arrows. Parameter values: $\alpha_{\text {post }}=0.01, \Delta I=-0.05, g_{\text {syn }}=0.00, k_{\text {post }}=0.002,0.0063$.

respectively) can be determined from (2.1):

$$
-\alpha_{\text {pre }}\left(I_{\text {pre }}-z_{\text {pre }}^{*}\right)-k_{\text {pre }} G\left(\Phi^{*}\right)=0 .
$$

Hence, according to (2.4) the value of phase locking mismatch, $\delta \Phi$, can be estimated as follows

$$
\delta \Phi=\Phi^{*}-\Phi_{c}=-\frac{1}{2 \pi} \arcsin \frac{\alpha_{p r e}\left(I_{p r e}-z_{p r e}^{*}\right)}{k_{p r e}} .
$$

The larger is the value of $k_{\text {pre }}$, the higher is the precision of phase locking. Notice, however, that if the feedback gain, $k_{\text {pre }}$, exceeds a critical threshold, the STDP phase locking regulatory mechanism described 

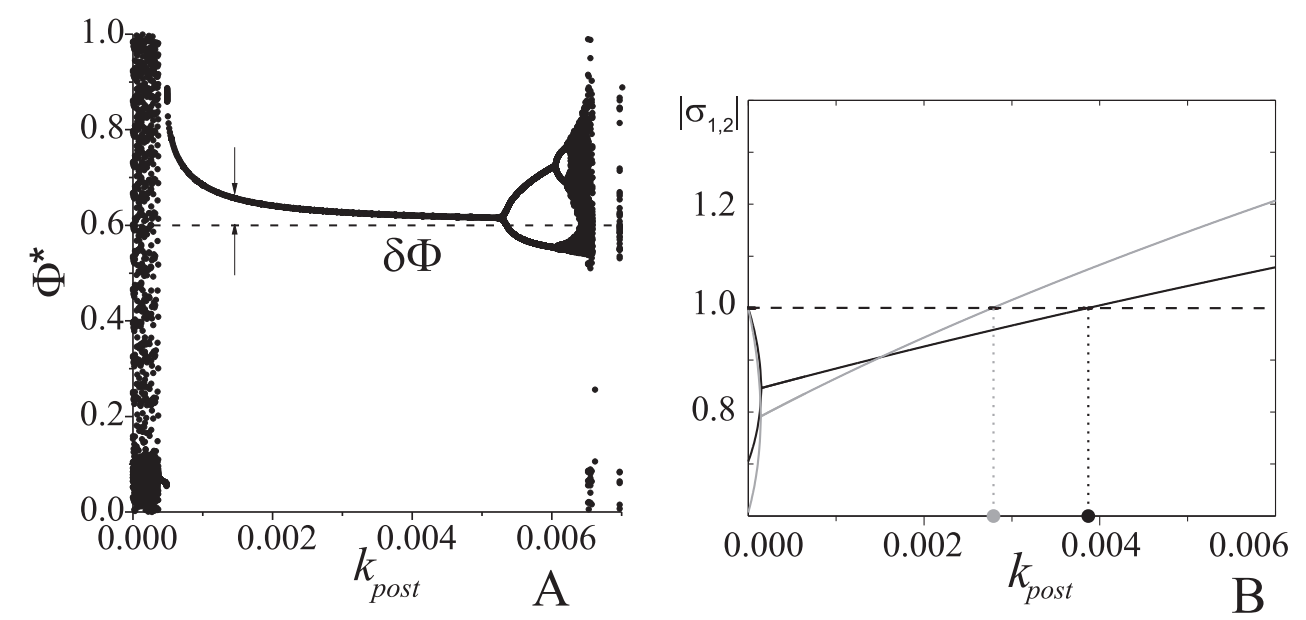

FiguRE 11. Bifurcation and stability diagrams for the case of postsynaptic control. Left panel: phase control bifurcation diagram. Values of the outcome phase $\Phi$ driven by Eqs. (2.2) versus feedback strength, $k_{\text {post }}$. Parameter values: $\alpha_{\text {post }}=0.01, \Delta I=$ $-0.05, g_{\text {syn }}=0.008, \Phi_{c}=0.6$. Right panel: A copy of Fig. 5 showing analytically derived stability diagram for this configuration. Notice that stability diagram in the right panel (derived analytically) is largely consistent with the bifurcation diagram in the left panel (obtained by means of numerical simulations). Slight inconsistencies are evident in the area where $k_{\text {post }}$ are small. These inconsistencies are due to that 1 ) our analytical derivations ignore the influence of synaptic coupling, $I_{s y n}$, and that 2) the fixed point may disappear when $k_{\text {post }}$ small.

above may fail. Loss of stability of the fixed point is a possible explanation for this observation. For extremely large values of $k_{\text {pre }}$ one can observe an "overregulation" catastrophe (Figure 12D). In short, STDP suppresses presynaptic neuron so hard that the neuron is eventually driven into excitable mode. This is shown in the upper panel of Fig. 12D. The value of $z_{p r e}$ exceeds the critical value, $I_{1}$ (see Fig. $4)$, and the presynaptic neuron becomes inhibited: no spikes are evoked.

In order see the range of parameters for which presynaptic STDP can be considered as a viable phase locking mechanism we calculated numerically dependence of $\Phi^{*}$ on $k_{\text {pre }}$ (Fig. 13). When $k_{\text {pre }}$ is small the relative phase $\Phi$ is not settling to a particular constant value; it "scans" through the whole interval of admissible values, [0,1). If $k_{\text {pre }}$ is increased beyond a threshold value the relative phase locks. Increasing the value of $k_{\text {pre }}$ further results in locking of relative phase in a neighborhood of the reference, $\Phi_{c}$, as predicted by (4.5).

With regards to the influence of STDP model (2.1) on behavior of the coupled system an interesting phenomenon can be observed: in-phase oscillations become apparently stable at some critical value of $k_{\text {pre }}$ (lower left corner of the plot). In other words, presynaptic STDP facilitates existing synaptic connections by providing synaptic efficacy equivalent to stronger synaptic coupling (transition from Fig. $6 \mathrm{~B}$ to Fig. $6 \mathrm{C}$ ). For larger values of $k_{\text {pre }}$ relative phase $\Phi$ jumps to a neighborhood of the reference phase $\Phi_{c}$. According to the figure, increments of $k_{\text {pre }}$ (in a relatively broad interval) result in improvements of the phase locking accuracy: relative phase $\Phi$ approaches $\Phi_{c}$ with the growth of $k_{\text {pre }}$. There is, however, a critical value of $k_{p r e}=k^{*}$ at which the fixed point becomes neutrally stable. Further increments of $k_{p r e}$ result in destabilization of the fixed point. 

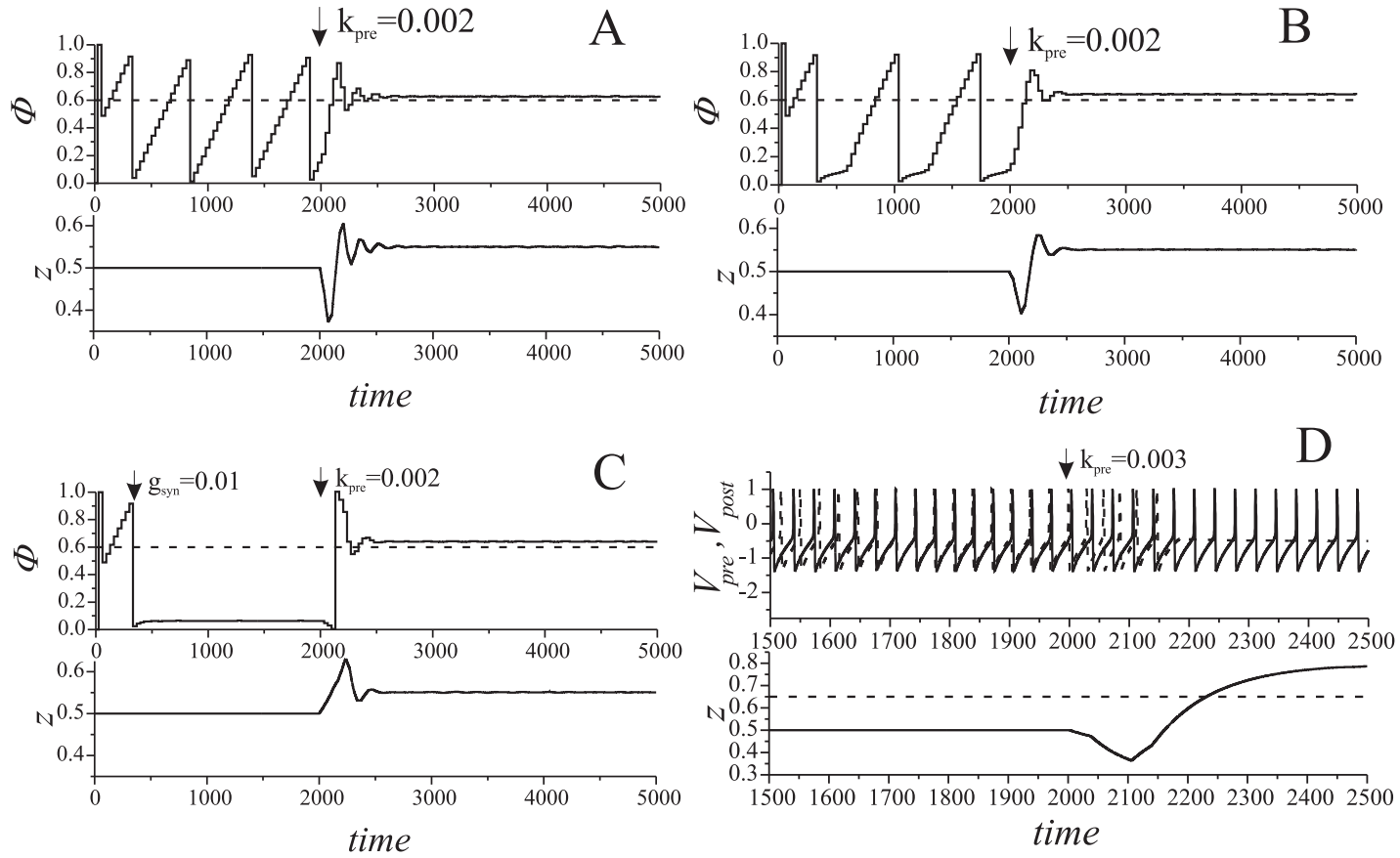

FiguRE 12. Dynamics of two neuronal oscillators with presynaptic control. Upper and the lower panels show the evolution of the relative phase shift (in A-C) and control variable, $z_{\text {pre }}$, respectively. A: No synaptic coupling. Parameter values: $\alpha_{\text {pre }}=0.01, k_{\text {pre }}=0.002, \Delta I=-0.05, g_{\text {syn }}=0, \Phi_{c}=0.6$. B: Phase pulling mode. Parameter values: $\alpha_{\text {pre }}=0.01, k_{\text {pre }}=0.002, \Delta I=-0.05, g_{\text {syn }}=0.008, \Phi_{c}=0.6 . \mathrm{C}$ : Switching the phase locking mode from the unsupervised mode (defined by the synaptic coupling) to the one enslaved by the reference phase. Parameter values: $\alpha_{\text {pre }}=$ $0.01, k_{\text {pre }}=0.002, \Delta I=-0.05, g_{\text {syn }}=0.01, \Phi_{c}=0.6$. D: Failure of the phase control due to overregulation effect. The upper panel shows membrane potentials in two neurons. Parameter values: $\alpha_{\text {pre }}=0.01, k_{\text {pre }}=0.003, \Delta I=-0.05, g_{\text {syn }}=0.008, \Phi_{c}=0.6$.

In order to assess stability of the relative phase dynamics we invoke the idea of spiking phase maps. Here the one-dimensional spiking phase map is extended as follows:

$$
T_{\text {pre }}:\left\{\begin{array}{l}
\Phi_{i} \rightarrow \Phi_{i+1}, \\
z_{\text {pre }}\left(t_{i}\right) \rightarrow z_{\text {pre }}\left(t_{i+1}\right), \quad i=1,2, \ldots \\
T_{\text {pre }}(i) \rightarrow T_{\text {pre }}(i+1) .
\end{array}\right.
$$

Variable $T_{\text {pre }}(i)$ is the period of presynaptic spikes; it is now time-varying due to the STDP feedback. Since there is a functional dependence between $\Phi_{i}$ and $T_{\text {pre }}(i)$, map (4.6) can be approximated by a two-dimensional one describing dynamics of the variables $\left(\Phi_{i}\left(T_{\text {pre }}(i)\right), z_{\text {pre }}\left(t_{i}\right)\right)$.

Investigating dynamics of $(2.8),(2.1),(2.4)$ numerically we have found that the critical gain $k^{*}$ corresponds to the neutral stability of $\Phi^{*}$ with zero real part of its complex conjugate multipliers. Therefore, Neimark-Saccer bifurcation takes place at $k_{p r e}=k^{*}[6]$. Figure 14 shows trajectories of the spiking phase map in the vicinity of $k^{*}$. One can see from this figure that if $k_{\text {pre }}<k^{*}$ then variables $\left(\Phi_{i}, z_{\text {pre }}\left(t_{i}\right)\right)$ travel towards the stable fixed point (see Figs. $14 \mathrm{~A}$ and $\mathrm{C}$ ). If, however, $k_{\text {pre }}>k^{*}$ then $\left(\Phi_{i}, z_{\text {pre }}\left(t_{i}\right)\right)$ move in the opposite direction (see Figs. $14 \mathrm{~B}$ and D), and the fixed point appears to be unstable. This behavior indicates that the bifurcation is subcritical (with positive first Lyapunov coefficient). Thus, for $k_{\text {pre }}>k^{*}$ relative phase $\Phi$ oscillates with a growing amplitude (Fig. $14 \mathrm{D}$ ). One can also observe that for $k_{\text {pre }}>k^{*}$, which are some distance apart from $k^{*}$, variable $z_{\text {pre }}$ (after a short transient) leaves the 


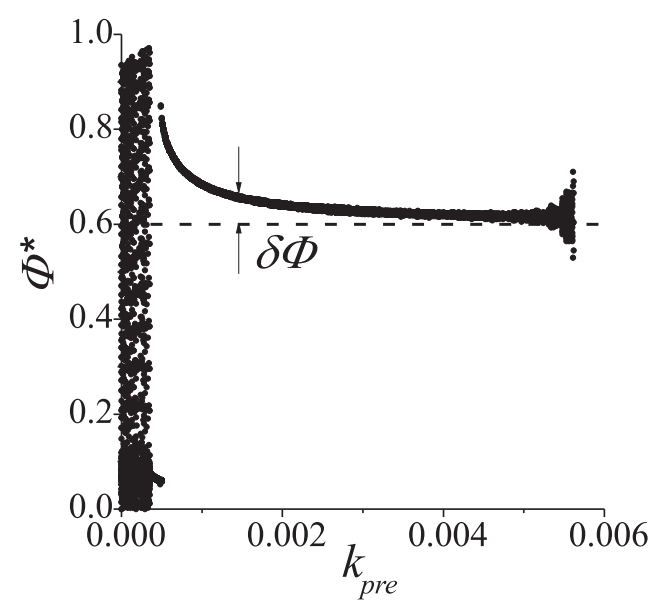

Figure 13. Phase control bifurcation diagram. Values of the outcome phase $\Phi$ driven by Eqs. (2.1) versus feedback strength, $k_{\text {pre }}$. Parameter values: $\alpha_{\text {pre }}=0.01, \Delta I=$ $-0.05, g_{\text {syn }}=0.008, \Phi_{c}=0.6$.
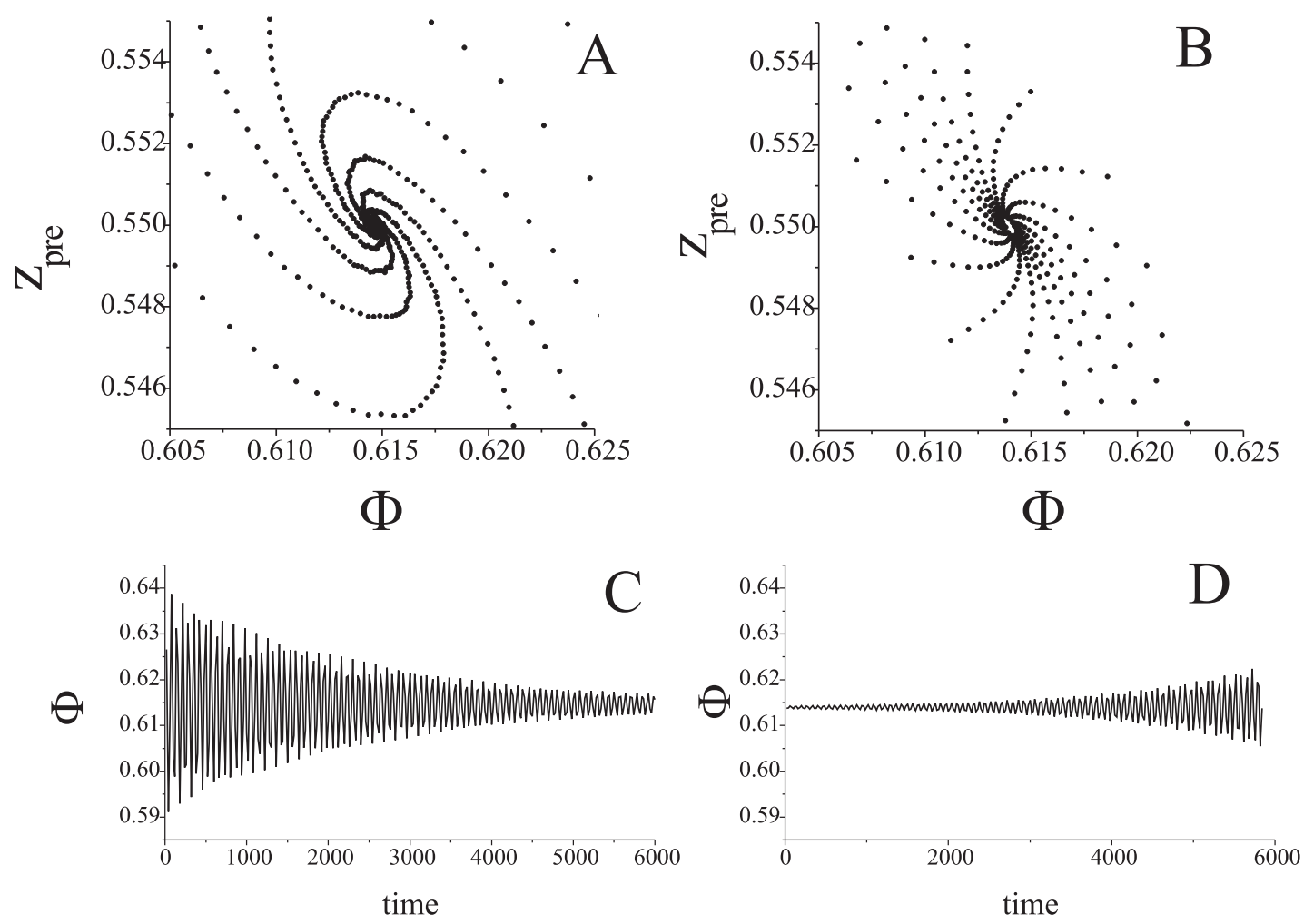

Figure 14. The dynamics of map (4.6) in the vicinity of the Neimark-Saccer bifurcation point, $k_{p r e}=k^{*}$. A and C. Phase plane dynamics and the oscillation profile near stable fixed point for $k_{p r e}=0.0054$. B and D. Phase plane dynamics and the oscillation profile near unstable fixed point for $k_{\text {pre }}=0.0057$. Parameter values: $\alpha_{\text {pre }}=0.01, \Delta I=$ $-0.05, g_{\text {syn }}=0.008, \Phi_{c}=0.6$. 
domain corresponding to the oscillatory mode (Fig. 4). This, in turn suppresses all oscillations in the presynaptic neuron.

In the bifurcation diagram in Fig. 13 a "cloud" of points emerges when $k_{\text {pre }}$ approaches the critical point $k^{*}$ from the left. The size of this cloud grows with $k_{\text {pre }}$ in a seemingly continuous way. This contrasts with our earlier remark about that the bifurcation is subcritical. Notice, however, that if $k_{\text {pre }}$ approaches $k^{*}$ from the left, real parts of the linearized map's eigenvalues are becoming negligibly small, and also the convergence rate to the fixed point is asymptotically decreasing to zero. Since numerical simulations were run over given and finite interval of time, the amplitude of this cloud, i.e. deviations of $\Phi$ from the fixed point at the end of the simulation, depends explicitly on the convergence rate of the map. The smaller is the convergence rate the higher are the chances that deviations of $\Phi$ from $\Phi_{c}$ are larger at the end of the simulation. This is exactly what we observe in the figure.

\subsection{Adaptive phase-locking STDP}

Let us finally investigate performance of the proposed spike-timing dependent adaptation law of the baseline variables $\lambda_{\text {post }}(3.28)$ :

$$
\begin{aligned}
\lambda_{\text {post }} & =\lambda_{\min }+\frac{\lambda_{\max }-\lambda_{\min }}{2}(1-\sin (\zeta)) \\
\dot{\zeta} & =\gamma\left|\Phi\left(t_{i}\right)-\Phi_{c}\right|, \gamma \in \mathbb{R}_{>0}, \lambda_{\max }>\lambda_{\min }, \lambda_{\max }, \lambda_{\min } \in \mathbb{R},
\end{aligned}
$$

According to Theorem 3.2 (see also $[26,27]$ ) such adaptation scheme ensures that $\lim _{i \rightarrow \infty} \Phi_{i}-\Phi_{c}=$ 0 provided that the value of $\gamma$ is sufficiently small and $\lambda_{\min }<\min _{\omega} \alpha_{\text {post }}\left(z_{\text {post }}^{*}(\omega)-I_{\text {post }}\right), \lambda_{\max }>$ $\max _{\omega} \alpha_{\text {post }}\left(z_{\text {post }}^{*}(\omega)-I_{\text {post }}\right)$. A very similar adaptation mechanism can be derived for $\lambda_{\text {pre }}$ as well by replacing subscripts post with pre in the above. Dynamics of adaptive phase-locking STDP in (2.8) with variable $z_{\text {post }}$ evolving according to $(2.2)$ is illustrated in Fig. 15. The figure confirms that when extracellular adaptation feedback is activated the error of phase locking is slowly vanishing with time.

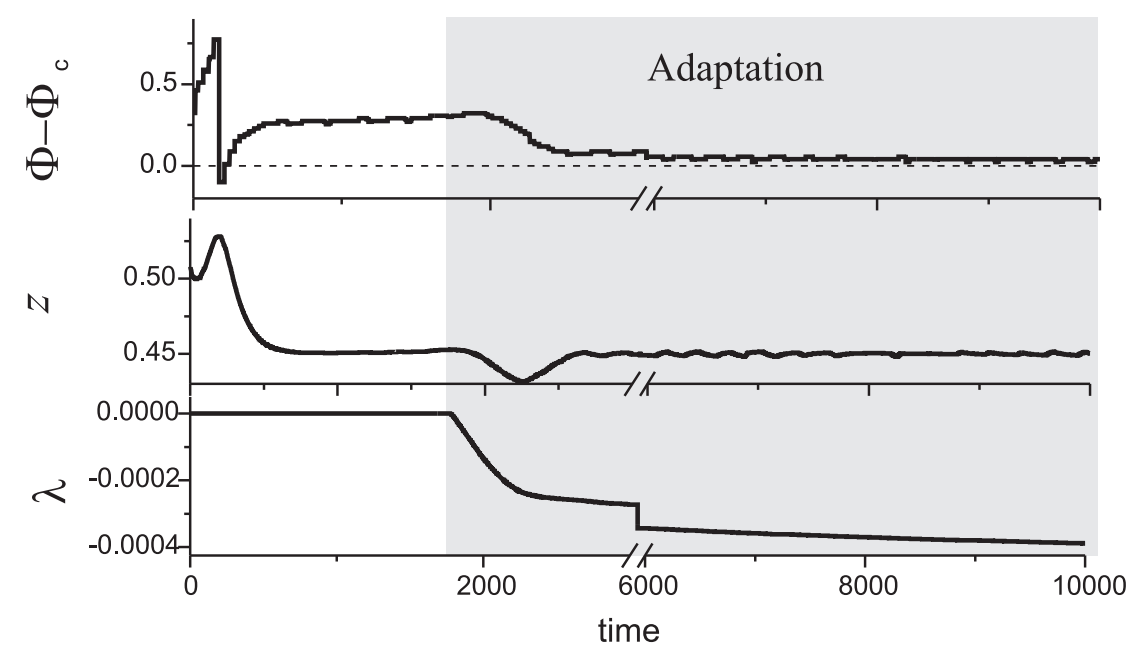

FIGURE 15. Adaptive compensation of phase locking errors via extracellular adaptation feedback. Illustration for the case of presynaptic control (2.1). Parameter values: $\alpha_{\text {pre }}=$ $0.01, \Delta I=-0.05, g_{\text {syn }}=0, \Phi_{c}=0.1, k_{\text {pre }}=0.0005, \gamma=0.001$. 


\subsection{Difference between Hodgkin-Huxley and Rowat-Selerston models. Effect of bistability}

Dynamics of the system in which individual oscillators are described by Hodgkin-Huxley equations remains qualitatively identical to the case of the Rowat-Selverston model (reduced model) if both oscillators operate in the oscillatory mode. The qualitative picture, however, changes when individual neurons (2.5) are no longer in the oscillatory regime but are functioning in the bistable mode in a vicinity of the saddle-node bifurcation point (Fig. 3). For the chosen set of model parameters, the interval of values of $\Delta I$ corresponding to the bistable mode in the Rowart-Selverston model is very narrow, and presence of such bistability doesn't appear to show significant influence on the dynamics of relative phase in the coupled system. With regards to the Hodgkin-Huxley equations, the interval of $\Delta I$ for which the model is bistable is substantially broader. In this case the inter-play between direct excitatory synaptic coupling and STDP driven depolarization mechanism can generate trajectories that are persistently "switching" between the limit cycle attractor and the fixed point (neuron resting potential). When the STDP mechanism is activated the spiking phase evolves along the corresponding PRC. As we have illustrated for the case of postsynaptic STDP (see, for example, Fig. 8) it may eventually reach the area (the gap in the figure) where the map itself is undefined. When this occurs the postsynaptic neuron becomes inhibited and its state begins to relax to the resting potential. Yet, due to the excitatory synaptic coupling, the state may be moved back to the domain corresponding to the oscillatory mode of the model. In this domain the system dynamics is again determined by the spiking phase map. Evidence and examples of such persistent transitions are shown in Fig. 16.
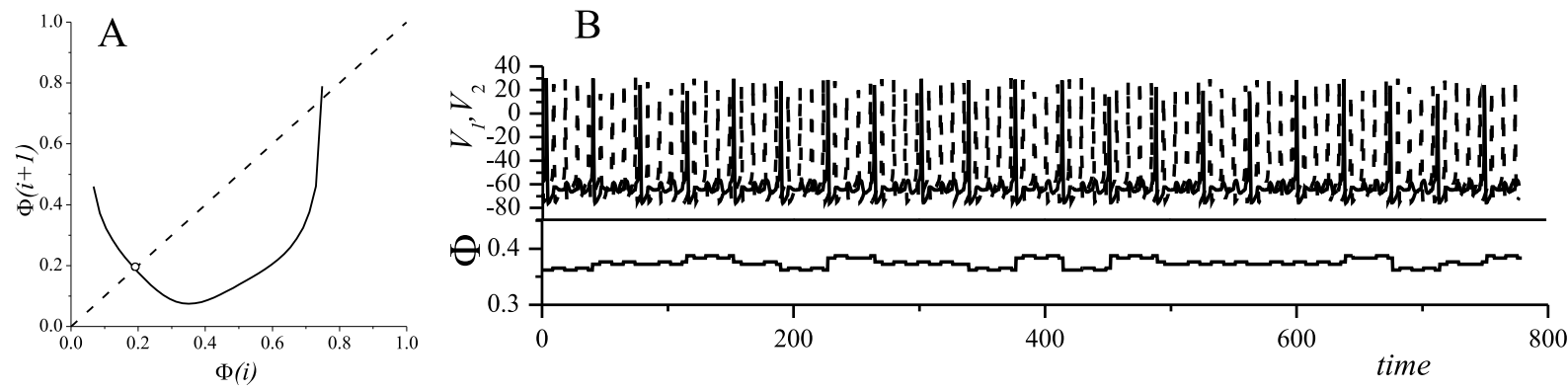

FIGURE 16. Interplay between direct synaptic coupling and postsynaptic STDP feedback in Hodgkin-Huxley neurons (2.5). A. Spiking phase map for the oscillatory mode defined in a certain interval of phase values. B. Trajectories of $V_{\text {pre }}$, in the presynaptic neuron (dashed curve), and $V_{\text {post }}$, postsynaptic response (solid curve), as functions of $t$. Time evolution of the oscillatory phase on modulus 1. Parameter values: $\Delta I=-1 \mu \mathrm{A} / \mathrm{cm}^{2}, g_{\text {syn }}=0.18 \mathrm{mS} / \mathrm{cm}^{2}, k_{\text {pre }}=0, k_{\text {post }}=0.5, \Phi_{c}=0.6$.

\section{Conclusion}

In this work we investigated viability of an STDP mechanism affecting neuronal excitability for the purposes of generating spiking patterns in a pair of coupled oscillators with broadly arbitrary phase lags between presynaptic and postsynaptic spikes. Theoretical analysis performed under the assumption that both coupled unites operate in the oscillatory mode predicted limitations (with regards to achievable phase lags of spiking) of non-adaptive couplings in the case of non-identical systems with different natural frequencies of oscillation. We have shown, however, that precise timing of signals in the system can still be achieved by means of a slow-varying spike-timing dependent self-tuning of the excitation parameters of the model. These predictions are confirmed in extensive numerical studies of the system when both 
oscillators are described either by biologically-plausible Hodgkin-Huxley equations or by their RowatSelverston reduction.

In addition to confirming plausibility of our theoretical findings, numerical analysis reveals rather complicated underlining dynamics of the coupled system. The range of observed phenomena for the pair of coupled neurons includes: overregulation catastrophe, route to chaos and substantial sensitivity to initial data and parameters when individual oscillators operate in the bistable mode. Observed variability of dynamical modes in such a simple system motivates further investigation in the subject which we hope to perform in future.

With respect to biological realism, our model is based on empirical observations that neuronal excitability may depend on spike-timing [24]; it is purely phenomenological and is, perhaps, too simple to be associated with a specific mechanism. It is therefore difficult to suggest a single experiment that would allow to validate the model reliably. Nevertheless, we believe that even without firm empirical justification the results can be used for enhancing of phase-locking of spikes in externally controlled neuronal cultures and animats. We would also like to notice that the resulting feedback circuits are quite similar by design to phase locked loop (PLL) control systems which are widely used in communication and engineering. This makes proposed regulatory STDP mechanisms readily implementable in artificial networks of electronic or cultured neurons for imitation modeling and design of neuromorphic and brain inspired information processing systems.

Acknowledgements. This research was supported in part by the Ministry of Education and Science of Russia (grant 14.B37.21.1073), by the Russian Foundation for Basic Research (grant 11-04-12144) and MCB Program of Russian Academy of Science.

\section{References}

[1] W.C. Abraham. Metaplasticity: tuning synapses and networks for plasticity. Nature Reviews Neuroscience, 9 (2008), 387-399.

[2] T. Bem, J. Rinzel. Short duty cycle destabilizes a half-center oscillator, but gap junctions can restabilize the anti-phase pattern. Journal of Neurophysiology, 91 (2004), 693-703.

[3] M.A. Diana, P. Bregestovski. Calcium and endocannabinoids in the modulation of inhibitory synaptic transmission. Cell Calcium, 37 (2005), 497-505.

[4] A. Dityatev, D.A. Rusakov. Molecular signals of plasticity at the tetrapartite synapse. Current Opinion in Neurbiology, 21 (2011), 353-359.

[5] W. Gerstner, R. Kempter, J.L. van Hemmen, H. Wagner. A neuronal learning rule for sub-millisecond temporal coding. Nature, 386 (1996), 76-78.

[6] J. Guckenheimer, P. Holmes. Nonlinear oscillations, dynamical systems, and bifurcations of vector fields. SpringerVerlag, 1986.

[7] S.Yu. Gordleeva, S.V. Stasenko, A.V. Semyanov, A.E. Dityatev, V.B. Kazantsev Bi-directional astrocytic regulation of neuronal activity within a network. Frontiers of Computational Neuroscience, 6 (2012), article 92.

[8] F.C. Hoppensteadt, E.M. Izhikevich. Weakly connected neural networks. Springer-Verlag, 1997.

[9] Y. Ikegaya, G. Aaron, R. Cossart, D. Aronov, I. Lampl, D. Ferster, R. Yuste. Synfire chains and cortical songs: Temporal modules of cortical activity. Science, 304 (2004), 559-564.

[10] E.M. Izhikevich. Polychronization: Computation with spikes. Neural Computation, 18 (2006), $245-282$.

[11] E.M. Izhikevich. Dynamical systems in neuroscience. The geometry of excitability and bursting. MIT Press, 2007.

[12] E.M. Izhikevich. Solving the distal reward problem through linkage of STDP and dopamine signaling. Cerebral Cortex, 17 (2007), 2443-2452.

[13] C. Kayser, M.A. Montemurro, N.K. Logothetis, S. Panzeri. Spike-phase coding boosts and stabilizes information carried by spatial and temporal spike patterns. Neuron, 61 (2009), 597-608.

[14] V. Kazantsev, I. Tyukin. Adaptive and phase selective spike timing dependent plasticity in synaptically coupled neuronal oscillators. PLOS ONE, 7 (2012), e30411.

[15] V. Kazantsev, S.Yu. Gordleeva, S.V. Stasenko, A.E. Dityatev. A homeostatic model of neuronal firing governed by feedback signals from extracellular matrix. PLOS ONE, 7 (2012), e41646.

[16] V.B. Kazantsev, V.I. Nekorkin, S. Binczak, S. Jacquir, J.M. Bilbault. Spiking dynamics of interacting oscillatory neurons. Chaos, 15 (2005), 023103.

[17] H.J. Koester, B. Sakmann. Calcium dynamics in single spines during coincident pre- and postsynaptic activity depend on relative timing of back-propagating action potentials and subthreshold excitatory postsynaptic potentials. Proc Natl Acad Sci USA, 95 (1998), 9596-9601. 
[18] F. Lanore, N. Rebola, M. Carta. Spike-timing-dependent plasticity induces presynaptic changes at immature hippocampal mossy fiber synapses. The Journal of Neuroscience, 29 (2009), 8299-8301.

[19] T. Ohno-Shosakua, Y. Hashimotodania, T. Maejima, M. Kano. Calcium signaling and synaptic modulation: Regulation of endocannabinoid-mediated synaptic modulation by calcium. Cell Calcium, 38 (2005), 369-374.

[20] A. Pikovsky, M. Rosenblum, J. Kurths. Synchronization: a unified concept in nonlinear sciences. Cambridge University Press, 2001.

[21] J.D. Rolston, S.M. Wagenaar, D.A.and Potter. Precisely timed spatiotemporal patterns of neural activity in dissociated cortical cultures. Neuroscience, 148 (2007), 294-303.

[22] P.F. Rowat, A.I. Selverston. Modeling the gastric mill central pattern generator with a relaxation-oscillator network. Journal of Neurophysiology, 70 (1993), 1030-1053.

[23] L.P. Shilnikov, A.L. Shilnikov, D.V. Turaev, L.O. Chua. Methods of qualitative theory in nonlinear dynamics. World Scientific, 2001.

[24] P.J. Sjostrom, E.A. Rancz, A. Roth, M. Hausser. Dendritic excitability and synaptic plasticity. Physiological Reviews, 88 (2008), 769-840.

[25] S. Song, K.D. Miller, L.F. Abbott. Competitive Hebbian learning through spike-timing-dependent synaptic plasticity. Nature Neuroscience, 3 (2000), 919-926.

[26] I. Tyukin. Adaptation in dynamical systems. Cambridge University Press, 2011.

[27] I. Tyukin, E. Steur, H. Neijmeijer, C. van Leeuwen. Small-gain theorems for systems with unstable invariant sets. SIAM Journal on Control and Optimization, 47 (2008), 849-882.

[28] A. Whitehead, M.I. Rabinovich, R. Huerta, V.P. Zhigulin, H.D.I. Abarbanel. Dynamical synaptic plasticity: a model and connection to some experiments. Biological Cybernetics, 88 (2003), 229-235. 